\title{
Linear Phase Paraunitary Filter Banks: Theory, Factorizations and Designs
}

\author{
Anand K. Soman, Member, IEEE, P. P. Vaidyanathan, Fellow, IEEE, and Truong Q. Nguyen, Member, IEEE
}

\begin{abstract}
M channel maximally decimated filter banks have been used in the past to decompose signals into subbands. The theory of perfect-reconstruction filter banks has also been studied extensively. Nonparaunitary systems with linear phase filters have also been designed. In this paper, we study paraunitary systems in which each individual filter in the analysis synthesis banks has linear phase. Specific instances of this problem have been addressed by other authors, and linear phase paraunitary systems have been shown to exist. This property is often desirable for several applications, particularly in image processing.

We begin by answering several theoretical questions pertaining to linear phase paraunitary systems. Next, we develop a minimal factorization for a large class of such systems. This factorization will be proved to be complete for even $M$. Further, we structurally impose the additional condition that the filters satisfy pairwise mirror-image symmetry in the frequency domain. This significantly reduces the number of parameters to be optimized in the design process. We then demonstrate the use of these filter banks in the generation of $M$-band orthonormal wavelets. Several design examples are also given to validate the theory.
\end{abstract}

\section{INTRODUCTION}

$\mathrm{D}$ IGITAL filter banks have been used in the past to decompose a signal into frequency subbands [1][12]. The signals in different subbands are then coded and transmitted. Such schemes are popular for encoding data from speech and image signals. The process of decomposition and eventual reconstruction are done by what is termed as the "analysis-synthesis" filter bank system shown in Fig. 1. In this scheme, the $H_{i}(z)$ are the analysis filters and $F_{i}(z)$ are the synthesis filters. The boxes with $\downarrow M$ denote the decimators, or the subsampling devices, whereas the boxes with $\uparrow M$ denote the expanders, which increase the sampling rate. Their definitions are as in [1], [3].

Fig. 2 is a representation of the subband coding scheme in terms of the polyphase matrices [3]. $\boldsymbol{E}(z)$ is the poly-

Manuscript received May 17, 1992; revised June 10, 1993. The Guest Editor coordinating the review of this paper and approving it for publication was Dr. Ahmed Tewfik. This was supported in part by the National Science Foundation under Grant MIP 8919196, in part by Tektronix Inc. and in part by Rockwell International.

A. K. Soman and P. P. Vaidyanathan are with the Department of Electrical Engineering, California Institute of Technology, Pasadena, CA 91125 .

T. Q. Nguyen is with the Lincoln Lab. Massachusetts Institute of Technology, Lexington, MA 02173

IEEE Log Number 9212190

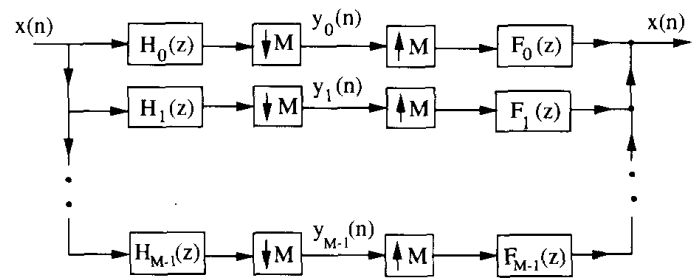

Fig. 1. A $M$-channel uniform filter bank.



Fig. 2. A filter bank drawn in terms of the polyphase matrices.

phase matrix corresponding to the analysis filters, and $\boldsymbol{R}(z)$ is the polyphase matrix corresponding to the synthesis filters. The decimators and expanders have been moved across the polyphase matrices using the noble identities [3]. It has been shown that it is indeed possible to perfectly reconstruct the original signal using such analysissynthesis systems [5]-[12]. In particular, this can be done by filters that have finite impulse response (FIR), and are hence guaranteed to be stable. One way to do this is to let $\boldsymbol{R}(z)=\boldsymbol{E}^{-1}(z)$, and then choose the matrix $\boldsymbol{E}(z)$ so that both matrices are FIR.

\section{A. Preliminaries}

Paraunitary systems: Another approach to design a perfect reconstruction system is to choose the matrix $\boldsymbol{E}(z)$ to be a FIR "paraunitary" matrix. A matrix is said to be paraunitary [8] if it satisfies the equation

$$
\tilde{E}(z) \boldsymbol{E}(z)=\boldsymbol{I}
$$

where $\tilde{\boldsymbol{E}}(z)=\boldsymbol{E}^{\dagger}\left(1 / z^{*}\right)$. The system can be guaranteed to have the perfect reconstruction property by having $\boldsymbol{R}(z)$ $=\tilde{E}(z)$. This paraunitary property can be traced back to classical network synthesis [14]. 
Consider the synthesis bank of Fig. 1. The original signal can be written in terms of the subband signals as

$$
x(n)=\sum_{k=0}^{M-1} \sum_{m} y_{k}(m) f_{k}(n-M m) .
$$

This can be viewed as a representation of the original signal in terms of a doubly indexed set of basis functions $\eta_{k m}(n)=f_{k}(n-M m)$. It is known [10], [13], [15] that this set of basis functions is orthonormal if and only if the polyphase matrix $\boldsymbol{R}(z)$ corresponding to these filters is paraunitary.

Another feature of the paraunitary analysis-synthesis system is that the analysis and synthesis filters are simply time-reversed conjugate versions of each other, and in particular therefore, they are of the same length.

Quantization: In a practical subband coding system, both the filter coefficients, as well as the subband signals are quantized. It has been shown [3], [16] that there exist structures which retain the paraunitary property inspite of coefficient quantization. The perfect-reconstruction property is however lost, when the signals in each subband are quantized. A paraunitary system still has some important features in the presence of subband quantization:

1) We can obtain bounds on the overall reconstruction error in terms of the quantization errors in each subband, no matter what the frequency responses of the filters are [17].

2) We are assured that the only error is due to signal quantization.

The coding gain [18] is often used as a criterion for judging the performance of these practical subband coding schemes.

$M$-band orthogonal wavelets: The relation between the $M$-channel paraunitary system and $M$-band orthogonal wavelets has been shown recently in [19], [20]. $M$-band wavelets have also been shown to provide a more compact representation of signals than the traditional binary wavelets [21]. The $M$-band wavelet is obtained by cascading the $M$-channel paraunitary system in a infinite tree-structure. Using a linear phase paraunitary system therefore gives us (with further conditions, see Section VI) an orthonormal basis of linear phase wavelets. This will be demonstrated later in this paper.

\section{B. Previous Work on Linear Phase Perfect Reconstruction Systems}

In several applications, and particularly in image coding, it is desirable to have each filter in the system to be a linear phase filter. This would not be necessary if there were no subband quantization, which is not a case of practical interest. The problem of designing two-channel linear phase, nonparaunitary, perfect reconstruction systems has been discussed in the past [22], [23]. However, for the two channel case, it can be shown that if a paraunitary system has linear phase filters, it is degenerate, i.e., the filters can be no better than a sum of two delays [3]. For $M$-channel paraunitary systems, linear phase property has been demonstrated in certain special cases, by Princen and Bradley in [24] and by Malvar in [25]. In [25], the author gives examples of linear phase Lapped Orthogonal Transforms (LOT), which have been shown to be order one paraunitary systems of a specific form. In [24] too, the filters mentioned correspond to a special type of paraunitary systems of order one. The more general case of linear phase paraunitary systems of larger degrees was addressed for the first time by Vetterli and Le Gall in [26]. The authors derive systems of higher degree from those of smaller degree by multiplication with certain types of paraunitary matrices, when the number of channels is even. For the four-channel case, the authors give judicious examples of such building blocks.

A structure is said to be minimal [10], if it uses the minimum number of delay elements to implement the particular transfer function. Completeness of a structure on the other hand implies being able to factorize a given linear phase paraunitary system in terms of the proposed structure. Another important consideration while designing filters by optimization is being able to characterize the building blocks in terms of a minimal number of free parameters. None of the earlier works addresses any of the above three issues. We shall address them in this paper. We will also present, for the first time, design examples of linear phase paraunitary systems of higher degrees.

\section{Aim of the Paper}

This paper attempts a thorough study of linear phase paraunitary filter banks. In particular, the following is the new contribution of this work: In Section II, we develop the theory of linear-phase paraunitary systems, and prove several new results. For the case where the number of channels $M$ is even, we present a factorization of the linear-phase paraunitary filter bank that is minimal as well as complete for a large class of filter banks important from a practical standpoint in Section III. In Section IV, we further structurally impose the constraint that the filters be pairwise symmetric around $\pi / 2$ in the frequency domain. This significantly reduces the number of variables to be optimized in the design. In Section V, we provide a cascade structure for linear-phase paraunitary systems when $M$ is odd, and prove that it is minimal. In Section VI, we apply the above ideas to generate symmetric, orthonormal, $M$-band wavelets. The issue of regularity [18], [12] is addressed. Finally in Section VII, we present some design examples of near-perfect reconstruction linear phase systems, based on formulating the filter bank design problem as a constrained optimization problem. Such a time-domain approach to filter bank design has also been proposed in [12].

\section{Notations}

Bold-faced quantities denote matrices and vectors, as in $\boldsymbol{A}$ and $\boldsymbol{x} \cdot \boldsymbol{A}^{T}, \boldsymbol{A}^{-1}$ and $\operatorname{Tr}(\boldsymbol{A})$ denote the transpose, the inverse, and the trace of the matrix $A$, respectively. A subscript on a matrix indicates its size, when the size is 
not clear from the context. Reserved symbols for special matrices are as follows: $\boldsymbol{I}$ is the identity matrix. The matrix $J_{N}$ is the antidiagonal matrix of size $N \times N$. For example, the antidiagonal matrix of size 4 is

$$
J_{4}=\left[\begin{array}{llll}
0 & 0 & 0 & 1 \\
0 & 0 & 1 & 0 \\
0 & 1 & 0 & 0 \\
1 & 0 & 0 & 0
\end{array}\right]
$$

0 will denote the null matrix, whose size will be clear from the context. $V_{K}$ will denote a special diagonal matrix of size $K \times K$, with alternating \pm 1 's on the diagonal, starting with +1 .

A superscript asterisk as in $f^{*}(n)$ denotes conjugation. Consider a transfer function $A(z)$. It can be written in terms of its $M$ polyphase components [27] as follows:

$$
\begin{aligned}
A(z)= & a_{0}\left(z^{M}\right)+z^{-1} a_{1}\left(z^{M}\right) \\
& +\cdots+z^{-(M-1)} a_{M-1}\left(z^{M}\right) .
\end{aligned}
$$

This is known as Type I polyphase. Let $H_{i}(z), i=0$, $\cdots, M-1$, be a set of analysis filters. They can be written as

$$
H_{k}(z)=\sum_{l=0}^{M-1} z^{-l} E_{k l}\left(z^{M}\right) \quad k=0, \cdots, M-1 .
$$

The matrix $\boldsymbol{E}(z)=\left[E_{k, l}(z)\right]$ is called the polyphase matrix of the analysis filters. A set of filters $H_{k}(z)$ whose polyphase matrix is paraunitary are said to form a paraunitary system (1.1). Throughout this paper, we will deal with real, causal, and FIR systems. Given such a system $\boldsymbol{E}(z)$ of $\operatorname{order} N$, we can write it explicitly as

$$
\begin{aligned}
\boldsymbol{E}(z)= & \boldsymbol{e}(0)+\boldsymbol{e}(1) z^{-1}+\boldsymbol{e}(2) z^{-2} \\
& +\cdots+\boldsymbol{e}(N) z^{-N}, \quad \boldsymbol{e}(N) \neq \mathbf{0}
\end{aligned}
$$

The analysis filters typically have order $M(N+1)-1$.

\section{Theory of Linear Phase Paraunitary Systems}

In order to obtain factorizations of linear phase paraunitary systems, we first need to obtain a characterization of their polyphase matrix which reflects the linear phase property of the individual filters. Consider a set of $M$ paraunitary transfer functions whose polyphase matrix $\boldsymbol{E}(z)$ satisfies the property [26]

$$
\boldsymbol{D} z^{-N} \boldsymbol{E}\left(z^{-1}\right) \boldsymbol{J}_{M}=\boldsymbol{E}(z)
$$

where $N$ is the order of the paraunitary matrix $\boldsymbol{E}(z)$. Such a polyphase matrix corresponds to a set of filters which have linear phase. The matrix $D$ is a diagonal matrix whose entries are \pm 1 's, the +1 's in those rows which correspond to symmetric filters and -1 's in those that correspond to antisymmetric filters. The filters described by this equation have the same center of symmetry $((N+$ 1) $M-1) / 2$.

It is conceivable that there are linear phase paraunitary systems which cannot be characterized as in (2.1). One example is that of the "delay chain," wherein the analysis filters are simply $H_{i}(z)=z^{-i}, i=0, \cdots, M-1$. However as said earlier, obtaining factorizations requires us to impose constraints on the polyphase matrix of the filters, and (2.1) represents a large class of filter banks important from a practical standpoint. In this paper, we will consider only those systems that can be described by (2.1). We will also show several good design examples based on such systems.

The linear phase constraint in conjunction with the paraunitary property imposes interesting conditions on the filters. The paraunitary property implies orthonormality of the impulse response to its own shifted versions [10], [15] and the linear phase property implies that the filters are time-reversed versions of themselves (upto a factor of \pm 1 ). This, for example, imposes a restriction on the length of the filters.

Fact 1: Let $F_{i}(z)$ be a set of $M$ linear phase paraunitary filters of length $L$ each with $f_{i}(0) \neq 0$. Then, $L \neq l M+$ 1 for any integer $l \geq 1$.

Proof: The orthonormality condition on the filters [10] in particular implies,

$$
\sum_{n=-\infty}^{\infty} f_{j}(n) f_{j}^{*}(n-l M)=\delta(i)
$$

If the length of the filters is $L=l M+1$, in view of linear phase property this means that

$$
f_{j}(0) f_{j}^{*}(0)=0
$$

implying that $f_{j}(0)=0$. Hence the length $L \neq l M+1$ for any integer $l \geq 1$.

The perfect reconstruction condition also imposes a constraint on the number of symmetric and antisymmetric functions in the filter bank. This is stated in the following theorem:

Theorem 1: Consider a $M$-channel linear phase perfect reconstruction system.

1) If $M$ is even, there are $M / 2$ symmetric, and $M / 2$ antisymmetric filters.

2) If $M$ is odd, there are $(M+1) / 2$ symmetric and $(M$ $-1) / 2$ antisymmetric filters.

This result has been proved in [28] for the special case where the order of the paraunitary matrix $\boldsymbol{E}(z)$ is one. The proof therein is based on subspace techniques, and moreover, does not extend to the case where $\boldsymbol{E}(z)$ has an arbitrary order. The result has been stated explicitly as an assumption in [26]. We provide below a formal proof that this is indeed true. Note, that the result is not restricted to paraunitary filter banks.

Proof: Consider (2.1). The trace of the matrix $\boldsymbol{D}$ holds the key to the number of symmetric and antisymmetric filters in the system. Using the fact that the matrix $E(z)$ is invertible, we have

$$
\begin{aligned}
\operatorname{Tr}(\boldsymbol{D}) & =\operatorname{Tr}\left(z^{N} \boldsymbol{E}(z) \boldsymbol{J}_{M} \boldsymbol{E}^{-1}\left(z^{-1}\right)\right) \\
& =\operatorname{Tr}\left(z^{N} \boldsymbol{E}^{-1}\left(z^{-1}\right) \boldsymbol{E}(z) \boldsymbol{J}_{M}\right) .
\end{aligned}
$$


We have used the fact that $\operatorname{Tr}(\boldsymbol{A B})=\operatorname{Tr}(\boldsymbol{B} \boldsymbol{A})$. The left hand side of this equation is constant. Hence its value can be found by evaluating the right hand side for one value of the variable $z$. Putting $z=1$ in the above equation we get,

$$
\operatorname{Tr}(\boldsymbol{D})=\operatorname{Tr}\left(\boldsymbol{E}^{-1}(1) \boldsymbol{E}(1) \boldsymbol{J}_{M}\right)=\operatorname{Tr}\left(\boldsymbol{J}_{M}\right)
$$

with the antidiagonal matrix $J_{M}$ as in (1.3). Therefore, it can be verified that $\operatorname{Tr}(\boldsymbol{D})=0$ if $M$ is even, and $\operatorname{Tr}(\boldsymbol{D})$ $=1$ if $M$ is odd. Hence there are an equal number of symmetric and antisymmetric functions if $M$ is even, whereas if $M$ is odd, there is one extra symmetric function.

In particular, the above theorem implies that all the filters cannot be zero phase. The proof of the above theorem also implies a interesting constraint on the order of the linear phase polyphase matrix $\boldsymbol{E}(z)$ when the number of filters $M$ is odd.

Corollary 1: If the number of channels $M$ is odd, the order $N$ of the polyphase matrix $\boldsymbol{E}(z)$ cannot be odd.

Proof: Consider (2.5), and let $N$ be odd. If one evaluates the right hand side of this equation at $z=-1$ instead of $z=1$, we get,

$$
\operatorname{Tr}(\boldsymbol{D})=\operatorname{Tr}\left((-1)^{N} \boldsymbol{E}^{-1}(-1) \boldsymbol{E}(-1) \boldsymbol{J}_{M}\right)=-\operatorname{Tr}\left(\boldsymbol{J}_{M}\right) .
$$

This, along with (2.6) would imply that $\operatorname{Tr}\left(J_{M}\right)=0$, but this is not possible since $M$ is odd. Hence we get a contradiction, proving that $N$ cannot be odd.

An interesting consequence of imposing the paraunitary constraint on an $M$-channel filter bank is that it guarantees that if the first $M-1$ filters are linear phase, the last filter is also linear phase. This is formally stated in the following theorem.

Theorem 2: Let a set of filters $F_{i}(z), i=0, \cdots, M$ - 1 be paraunitary, and let the first $M-1$ of them have linear phase. Then the last one is guaranteed to have linear phase.

Before we prove the theorem we will prove a lemma which will help us in the proof.

Lemma 1: If $M-1$ functions of a FIR paraunitary system are known, the last one is uniquely determined (upto a factor of the form $\left.\left(e^{j \theta}\right) z^{I M}\right)$.

Proof: Let $F_{i}(z), i=0, \cdots, M-1$ form a FIR paraunitary system. Let, if possible, $u(z)$ be another FIR function, which along with $F_{i}(z), i=0, \cdots, M-2$ forms a paraunitary system. Let $\boldsymbol{E}^{\prime}(z)$, the polyphase matrix corresponding to this modified set of paraunitary functions, be partitioned as

$$
\boldsymbol{E}^{\prime}(z)=\left[\begin{array}{c}
\boldsymbol{E}_{1}^{\prime}(z) \\
\boldsymbol{u}(z)
\end{array}\right] .
$$

This means that the row vector $\boldsymbol{u}(z)$ has as its elements the polyphase components of the filter $u(z)$. Since $\boldsymbol{E}^{\prime}(z)$ is unitary on the unit circle, $\boldsymbol{u}\left(e^{j \omega}\right)$ is uniquely determined upto a scale factor of the form $e^{j \Phi(\omega)}$. Hence, by analytic continuation, $u(z)=A\left(z^{M}\right) F_{M-1}(z)$, where $A(z)$ is all pass. It can be verified that the condition $\operatorname{det}\left(\boldsymbol{E}^{\prime}(z)\right)=$ delay, which is necessary for paraunitariness, implies that $A(z)=\left(e^{j \theta}\right) z^{l M}$. Hence, given $M-1$ functions of an FIR paraunitary system, the last function is determined upto a factor of the form $\left(e^{j \theta}\right) z^{l M}$.

Using this lemma, we can now prove Theorem 2 .

Proof of Theorem 2: Let $\boldsymbol{E}(z)$ be a paraunitary polyphase matrix corresponding to a set of filters that have linear phase. Let $E_{1}(z)$ be the polyphase matrix of size ( $M$ - 1) $\times M$ corresponding to the first $M-1$ filters in the system, and let $\boldsymbol{u}(z)$ be the row vector whose elements are the polyphase components corresponding to the last filter $u(z)$ of the system. Now, (2.1) can be rewritten as

$$
D z^{-n}\left[\begin{array}{c}
\boldsymbol{E}_{1}\left(z^{-1}\right) \\
\boldsymbol{u}\left(z^{-1}\right)
\end{array}\right] \boldsymbol{J}_{M}=\left[\begin{array}{c}
\boldsymbol{E}_{1}(z) \\
\boldsymbol{v}(z)
\end{array}\right]
$$

This means that the row vector $v(z)$ has as its element the polyphase components of the filter $v(z)$, which is the time-reversed version of $u(z)$ (upto \pm 1 ). Now, since all matrices on the left hand side of this equation are paraunitary, the matrix on the right hand side of this equation is also paraunitary. But the first block of this matrix is $\boldsymbol{E}_{1}(z)$. This means by Lemma 1 , that $v(z)= \pm z^{l M} u(z)$. But since $v(z)$ is also the time-reversed version of the filter $u(z)$, it implies that $u(z)$ has linear phase.

\section{Factorization of Linear Phase Paraunitary SySTEMS FOR EVEN $M$}

In this section, we will first derive a cascade-form structure for synthesizing linear phase paraunitary systems. Our theory will provide an interpretation for the condition mentioned in [26]. We will then prove the main result of this section, namely, every linear phase paraunitary system described by (2.1) can be factored in terms of the proposed structure.

The synthesis procedure consists of two steps. In the first step, we propagate the property that the set of filters generated be pairwise time-reversed versions of one another. This means that they are related as $h_{k}^{\prime}(n)=$ $h_{M-1-k}^{\prime}(n), k=0, \cdots, M-1$.

Notice that the sum of two sequences related as above is symmetric, and their difference is antisymmetric. Furthermore, any linear combination of symmetric (antisymmetric) sequences is symmetric (antisymmetric). In the second step, we add an orthogonal block which performs these operations on the pairwise symmetric sequences to obtain filters that have linear phase.

The reason for this two step approach is that, it can be shown that it is not possible to propagate the linear-phase property itself by addition of further building blocks.

Consider Fig. 3. The pairwise time-reversed property implies the following relation between the filters:

$$
\begin{array}{r}
H_{m, M-1-k}^{\prime}(z)=z^{-((m+1) M-1)} H_{m, k}^{\prime}\left(z^{-1}\right), \\
k=0, \cdots, L-1
\end{array}
$$




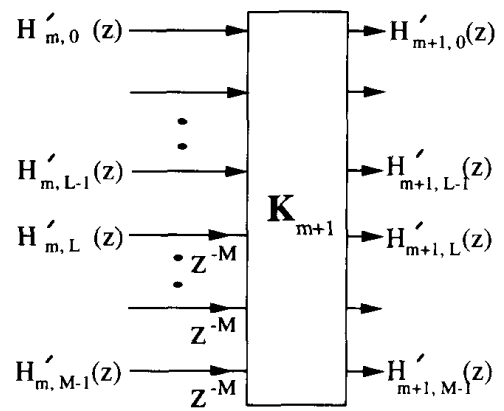

Fig. 3. One stage of the filter bank developed in Section III

where $L=M / 2$. If $\boldsymbol{F}_{m}(z)$ is the polyphase matrix corresponding to these filters, then

$$
z^{-m} \boldsymbol{J}_{M} \boldsymbol{F}_{m}\left(z^{-1}\right) \boldsymbol{J}_{M}=\boldsymbol{F}_{m}(z) \text {. }
$$

Consider Fig. 3 and let

$$
\boldsymbol{F}_{m+1}(z)=\boldsymbol{K}_{m+1} \boldsymbol{\Lambda}(z) \boldsymbol{F}_{m}(z)
$$

where $\boldsymbol{\Lambda}(z)=\left(\begin{array}{cc}\boldsymbol{I}_{\boldsymbol{M}} / 2 & \mathbf{0} \\ \mathbf{0} & z^{-1} \boldsymbol{I}_{M / 2}\end{array}\right)$, and $\boldsymbol{F}_{m+1}(z)$ is the polyphase matrix corresponding to the filters at the next stage. If $\boldsymbol{K}_{m+1}$ is an orthogonal matrix, we have

$$
\boldsymbol{F}_{m}(z)=\boldsymbol{\Lambda}\left(z^{-1}\right) \boldsymbol{K}_{m+1}^{T} \boldsymbol{F}_{m+1}(z) .
$$

For the filters at the next stage to retain the pairwise timereversed property, we need

$$
z^{-(m+1)} \boldsymbol{J}_{M} \boldsymbol{F}_{m+1}\left(z^{-1}\right) \boldsymbol{J}_{M}=\boldsymbol{F}_{m+1}(z) .
$$

Using (3.4) in (3.2), we obtain the following equation:

$$
\begin{gathered}
z^{-m} \boldsymbol{J}_{M} \boldsymbol{\Lambda}(z) \boldsymbol{K}_{m+1}^{T} \boldsymbol{F}_{m+1}\left(z^{-1}\right) \boldsymbol{J}_{M} \\
=\boldsymbol{\Lambda}\left(z^{-1}\right) \boldsymbol{K}_{m+1}^{T} \boldsymbol{F}_{m+1}(z) .
\end{gathered}
$$

By imposing (3.5), and using the identity $\Lambda(z) \boldsymbol{J}_{M} \boldsymbol{\Lambda}(z)=$ $z^{-1} J_{M}$, we see that the necessary and sufficient condition on $\boldsymbol{K}_{m+1}$ is, $\boldsymbol{K}_{m+1} \boldsymbol{J}_{M} \boldsymbol{K}_{m+1}^{T}=\boldsymbol{J}_{M}$. By partitioning $\boldsymbol{K}_{m+1}$ as $\left(\begin{array}{ll}A_{B^{\prime}} & { }_{D}^{\prime} \\ D^{\prime}\end{array}\right)$, we can verify that the necessary and sufficient condition for (3.5) to hold is that the matrix $\boldsymbol{K}_{m+1}$ be of the form

$$
\boldsymbol{K}_{m+1}=\left(\begin{array}{cc}
\boldsymbol{A}^{\prime} & \boldsymbol{C}^{\prime} \\
\boldsymbol{J}_{M / 2} \boldsymbol{C}^{\prime} \boldsymbol{J}_{M / 2} & \boldsymbol{J}_{M / 2} \boldsymbol{A}^{\prime} \boldsymbol{J}_{M / 2}
\end{array}\right) .
$$

Thus, $\boldsymbol{K}_{m+1}$ can be rewritten as

$$
\boldsymbol{K}_{m+1}=\left(\begin{array}{cc}
\boldsymbol{I}_{M / 2} & \mathbf{0} \\
\mathbf{0} & \boldsymbol{J}_{M / 2}
\end{array}\right)\left(\begin{array}{ll}
\boldsymbol{A}_{m+1} & \boldsymbol{C}_{m+1} \\
\boldsymbol{C}_{m+1} & \boldsymbol{A}_{m+1}
\end{array}\right)\left(\begin{array}{cc}
\boldsymbol{I}_{M / 2} & \mathbf{0} \\
\mathbf{0} & \boldsymbol{J}_{M / 2}
\end{array}\right)
$$

where $\boldsymbol{A}_{m+1}=\boldsymbol{A}^{\prime}$, and $\boldsymbol{C}_{m+1}=\boldsymbol{C}^{\prime} \boldsymbol{J}_{M / 2}$.

It can be verified that a matrix $K_{0}$ with a form similar to that described above can be used to initialize the process.

Given a paraunitary polyphase matrix $\boldsymbol{F}(z)$ of order $N$, corresponding to a set of filters that are pair-wise timereversed versions of one another i.e.,

$$
z^{-N} \boldsymbol{J}_{M} \boldsymbol{F}\left(z^{-1}\right) \boldsymbol{J}_{M}=\boldsymbol{F}(z)
$$

we are interested in obtaining a paraunitary polyphase matrix $\boldsymbol{E}(z)$ of order $N$ corresponding to a set of linear phase paraunitary filters, i.e., satisfying (2.1). Let $\boldsymbol{E}(z)$ $=\boldsymbol{S} \boldsymbol{F}(z)$, where $\boldsymbol{S}$ is a orthogonal matrix. Under the constraint (3.8), it can be shown (by substitution and simplification) that $\boldsymbol{E}(z)$ satisfies (2.1) if and only if $\boldsymbol{S}^{T} \boldsymbol{D S}=$ $J_{M}$.

Hence, the following product gives linear phase paraunitary polyphase matrix:

$$
\boldsymbol{E}(z)=\boldsymbol{S P T _ { N }} \boldsymbol{P \Lambda}(z) \boldsymbol{P T _ { N - 1 }} \boldsymbol{P \Lambda}(z) \boldsymbol{P} \cdots \boldsymbol{P \Lambda}(z) \boldsymbol{P} \boldsymbol{T}_{0} \boldsymbol{P}
$$

where

$$
\boldsymbol{P}=\left(\begin{array}{cc}
\boldsymbol{I}_{M / 2} & \mathbf{0} \\
\mathbf{0} & \boldsymbol{J}_{M / 2}
\end{array}\right) \text { and } \boldsymbol{T}_{i}=\left(\begin{array}{cc}
\boldsymbol{A}_{i} & \boldsymbol{C}_{i} \\
\boldsymbol{C}_{i} & \boldsymbol{A}_{i}
\end{array}\right) .
$$

Noting that $\boldsymbol{P} \boldsymbol{\Lambda}(z) \boldsymbol{P}=\boldsymbol{\Lambda}(z)$, we obtain

$$
\boldsymbol{E}(z)=\boldsymbol{S P T}_{N} \mathbf{\Lambda}(z) \boldsymbol{T}_{N-1} \boldsymbol{\Lambda}(z) \cdots \boldsymbol{\Lambda}(z) \boldsymbol{T}_{0} \boldsymbol{P}
$$

which is shown in Fig. 4.

Minimal Characterization: The matrix $\boldsymbol{K}_{m+1}$ is parameterized completely by parametrizing all orthogonal matrices of the form $\boldsymbol{T}_{m+1}=\left(\begin{array}{cc}\boldsymbol{A}_{m+1} & \boldsymbol{C}_{m+1} \\ \boldsymbol{A}_{m+1}\end{array}\right)$. Now, such a matrix can always be factored as

$$
\begin{aligned}
\left(\begin{array}{ll}
\boldsymbol{A}_{m+1} & \boldsymbol{C}_{m+1} \\
\boldsymbol{C}_{m+1} & \boldsymbol{A}_{m+1}
\end{array}\right)= & \left(\begin{array}{cc}
\boldsymbol{I}_{M / 2} & \boldsymbol{I}_{M / 2} \\
\boldsymbol{I}_{M / 2} & -\boldsymbol{I}_{M / 2}
\end{array}\right)\left(\begin{array}{ll}
\boldsymbol{W} & \mathbf{0} \\
\mathbf{0} & \boldsymbol{U}
\end{array}\right) \\
& \cdot\left(\begin{array}{rr}
\boldsymbol{I}_{M / 2} & \boldsymbol{I}_{M / 2} \\
\boldsymbol{I}_{M / 2} & -\boldsymbol{I}_{M / 2}
\end{array}\right)
\end{aligned}
$$

where $\boldsymbol{W}=\left(\boldsymbol{A}_{m+1}+\boldsymbol{C}_{m+1}\right) / 2$ and $\boldsymbol{U}=\left(\boldsymbol{A}_{m+1}-\right.$ $\left.C_{m+1}\right) / 2$. Thus $\boldsymbol{T}_{m+1}$ is orthogonal if and only if the two matrices $\boldsymbol{W}$ and $U$ are orthogonal. The orthogonal matrices $W$ and $U$ can be completely characterized by $\left(\begin{array}{c}M / 2 \\ 2\end{array}\right)$ rotations each [29].

On the other hand, it can be shown that a unitary matrix $\boldsymbol{S}$ satisfies the condition $\boldsymbol{S}^{T} \boldsymbol{D S}=J_{M}$ if and only if it can be written as

$$
\boldsymbol{S}=(1 / \sqrt{ } 2)\left(\begin{array}{cc}
S_{0} & 0 \\
\mathbf{0} & \boldsymbol{S}_{1}
\end{array}\right)\left(\begin{array}{cc}
\boldsymbol{I}_{M / 2} & \boldsymbol{J}_{M / 2} \\
\boldsymbol{I}_{M / 2} & -\boldsymbol{J}_{M / 2}
\end{array}\right)
$$

where $S_{0}$ and $S_{1}$ are orthogonal matrices of size $M / 2 \times$ $M / 2$ (Partition $S$ into four blocks, sustitute in $S^{T} D S=$ $J_{M}$ and simplify). $S$ can hence be parameterized by $2\left({ }_{2}^{M / 2}\right)$ rotations.

We now come to the main result of this section, which is the converse of the previous result.

Theorem 3: Let $\boldsymbol{E}(z)$ be a FIR linear phase paraunitary matrix, satisfying (2.1). Then it can always be factored as in (3.11), where $\boldsymbol{\Lambda}(z)=\left(\begin{array}{ll}I_{M / 2} & { }^{0} \\ z^{-1} I_{M / 2}\end{array}\right)$, and $\boldsymbol{T}_{i}$ and $\boldsymbol{P}$ are as in (3.10).

Proof: The rest of this section deals with the proof of the above theorem. The reader may skip over to the next section without loss of continuity.

The proof of the theorem will use the definition of "balanced vectors"' which we now propose: 




Fig. 4. An equivalent structure for the linear phase paraunitary system.

Definition: A vector $y$ is said to be "balanced" if it is orthogonal to its own flipped version, i.e., it satisfies the equation

$$
\boldsymbol{y}^{T} \boldsymbol{J}_{M} \boldsymbol{y}=0 .
$$

The significance of balanced vectors has been explained in Appendix A.

Proof of the Theorem: In this case we are given a matrix $\boldsymbol{E}(z)$ satisfying (2.1). The first step is to show that from this linear phase paraunitary matrix, we can always get a polyphase matrix $\boldsymbol{F}(z)$ whose filters are pairwise time-reversed versions of one another [satisfying (3.8)]. For this, let $S$ be any matrix of the form given in (3.13), where $S_{0}$ and $S_{1}$ are arbitrary orthogonal matrices. Then it can be shown by substitution that the product $\boldsymbol{F}(z)=$ $\boldsymbol{S}^{T} \boldsymbol{E}(z)$ satisfies (3.8).

Now we need to show that the matrix $F(z)$ can always be factored into the required form. This is achieved by performing the "order-reduction" process as outlined below. Let

$$
\begin{gathered}
\boldsymbol{F}_{m+1}(z)=f_{m+1}(0)+f_{m+1}(1) z^{-1}+f_{m+1}(2) z^{-2} \\
+\cdots+f_{m+1}(m+1) z^{-(m+1)} \\
f_{m+1}(m+1) \neq 0 .
\end{gathered}
$$

We will show that there exists $\boldsymbol{F}_{m}(z)$ of the form

$$
\begin{aligned}
\boldsymbol{F}_{m}(z)= & \boldsymbol{f}_{m}(0)+\boldsymbol{f}_{m}(1) z^{-1}+\boldsymbol{f}_{m}(2) z^{-2} \\
& +\cdots+\boldsymbol{f}_{m}(m) z^{-m}, \quad \boldsymbol{f}_{m}(m) \neq \mathbf{0}
\end{aligned}
$$

and satisfying the required properties. Let $\boldsymbol{F}_{m+1}(z)$ satisfy (3.5). Specifically, we will now show that it can always be written as

$$
\boldsymbol{F}_{m+1}(z)=\boldsymbol{P} \boldsymbol{T}_{m+1} \boldsymbol{P} \boldsymbol{\Lambda}(z) \boldsymbol{F}_{m}(z)
$$

where $\boldsymbol{F}_{m}(z)$ satisfies (3.2), and the matrices $\boldsymbol{P}, \boldsymbol{T}_{m+1}$ and $\boldsymbol{\Lambda}(z)$ have the form described earlier. Paraunitariness of $\boldsymbol{F}_{m}(z)$ follows by noting that

$$
\boldsymbol{F}_{m}(z)=\boldsymbol{\Lambda}\left(z^{-1}\right) \boldsymbol{P} \boldsymbol{T}_{m+1}^{T} \boldsymbol{P} \boldsymbol{F}_{m+1}(z)
$$

where all matrices on the right hand side of this equation are paraunitary.

Linear phase property: We want to show that $\boldsymbol{F}_{m}(z)$ satisfies (3.2). Substituting (3.16) into (3.5), we get

$$
\begin{gathered}
z^{-(m+1)} \boldsymbol{J}_{M} \boldsymbol{P} \boldsymbol{T}_{m+1} \boldsymbol{P} \boldsymbol{\Lambda}\left(z^{-1}\right) \boldsymbol{F}_{m}\left(z^{-1}\right) \boldsymbol{J}_{M} \\
=\boldsymbol{P} \boldsymbol{T}_{m+1} \boldsymbol{P} \boldsymbol{\Lambda}(z) \boldsymbol{F}_{m}(z) .
\end{gathered}
$$

Since $\boldsymbol{P}^{-1}=\boldsymbol{P}$ and $\boldsymbol{F}_{m}(z)$ is paraunitary, we get

$$
\begin{array}{r}
z^{-(m+1)} \boldsymbol{\Lambda}\left(z^{-1}\right) \boldsymbol{F}_{m}\left(z^{-1}\right) \boldsymbol{J}_{M} \tilde{\boldsymbol{F}}_{m}(z) \\
=\boldsymbol{P} \boldsymbol{T}_{m+1}^{T} \boldsymbol{P} \boldsymbol{J}_{M} \boldsymbol{P} \boldsymbol{T}_{m+1} \boldsymbol{P} \boldsymbol{\Lambda}(z) .
\end{array}
$$

If $\boldsymbol{T}_{m+1}$ is an orthogonal matrix of the form described in (3.10), and $\boldsymbol{P}$ has the form described in (3.10), then it can be verified that $\boldsymbol{P} \boldsymbol{T}_{m+1}^{T} \boldsymbol{P J _ { M }} \boldsymbol{P} \boldsymbol{T}_{m+1} \boldsymbol{P}=\boldsymbol{J}_{M}$. Hence, we get

$$
\begin{aligned}
& \quad z^{-(m+1)} \boldsymbol{\Lambda}\left(z^{-1}\right) \boldsymbol{F}_{m}\left(z^{-1}\right) \boldsymbol{J}_{M} \tilde{\boldsymbol{F}}_{m}(z)=\boldsymbol{J}_{M} \mathbf{\Lambda}(z) \\
& \text { i.e., } \\
& \qquad z^{-m}\left[z^{-1} \boldsymbol{\Lambda}\left(z^{-1}\right) \boldsymbol{J}_{M} \mathbf{\Lambda}\left(z^{-1}\right)\right] \boldsymbol{F}_{m}\left(z^{-1}\right) \boldsymbol{J}_{M} \tilde{\boldsymbol{F}}_{m}(z)=\boldsymbol{I} .
\end{aligned}
$$

It can be verified that $\left[z^{-1} \Lambda\left(z^{-1}\right) J_{M} \Lambda\left(z^{-1}\right)\right]=J_{M}$. Substituting this into (3.21) and rearranging the terms, we get (3.2).

Causality: It only remains to show that there exists a matrix $\boldsymbol{T}_{m+1}$ such that $\boldsymbol{F}_{m}(z)$ obtained from (3.17) is causal. Both the linear phase property, and the paraunitary property continue to hold for the reduced system as long as the matrix $T_{m+1}$, is any orthogonal matrix of the required form (3.10). Indeed, it is the causality condition on the reduced system which determines the particular choice of the matrix $\boldsymbol{T}_{m+1}$.

From (3.17) we get,

$$
\begin{aligned}
\boldsymbol{F}_{m}(z)= & \left(\begin{array}{cc}
\boldsymbol{I}_{M / 2} & \mathbf{0} \\
\mathbf{0} & \mathbf{0}
\end{array}\right) \boldsymbol{P} \boldsymbol{T}_{m+1}^{T} \boldsymbol{P \boldsymbol { F } _ { m + 1 } ( z )} \\
& +\left(\begin{array}{cc}
\mathbf{0} & \mathbf{0} \\
\mathbf{0} & z \boldsymbol{I}_{M / 2}
\end{array}\right) \boldsymbol{P} \boldsymbol{T}_{m+1}^{T} \boldsymbol{P} \boldsymbol{F}_{m+1}(z)
\end{aligned}
$$

The second term on the right hand side of this equation is responsible for the noncausality. In particular, the noncausal part of the second term is given by

$$
\left(\begin{array}{cc}
\mathbf{0} & \mathbf{0} \\
\mathbf{0} & z \boldsymbol{I}_{M / 2}
\end{array}\right) \boldsymbol{P} \boldsymbol{T}_{m+1}^{T} \boldsymbol{P} \boldsymbol{f}_{m+1}(0) \text {. }
$$

We have to show that there exists a matrix $\boldsymbol{T}_{\boldsymbol{m}+1}$ of the form in (3.10) which makes this term equal to zero. Let

$$
\boldsymbol{T}_{m+1}=\left(\begin{array}{ll}
\boldsymbol{A}_{m+1} & \boldsymbol{C}_{m+1} \\
\boldsymbol{C}_{m+1} & \boldsymbol{A}_{m+1}
\end{array}\right) .
$$


Simplifying (3.23), we find that $\boldsymbol{T}_{m+1}$ should be such that

$$
\left(\begin{array}{cc}
\mathbf{0} & \mathbf{0} \\
\mathbf{0} & \boldsymbol{J}_{M / 2}
\end{array}\right)\left(\begin{array}{ll}
\boldsymbol{A}_{m+1}^{T} & \boldsymbol{C}_{m+1}^{T} \boldsymbol{J}_{M / 2} \\
\boldsymbol{C}_{m+1}^{T} & \boldsymbol{A}_{m+1}^{T} \boldsymbol{J}_{M / 2}
\end{array}\right) \boldsymbol{f}_{m+1}(0)=\mathbf{0} .
$$

Hence, it is sufficient to find $\boldsymbol{A}_{m+1}$ and $\boldsymbol{C}_{m+1}$ such that

$$
\left(C_{m+1}^{T} \quad A_{m+1}^{T} J_{M / 2}\right) f_{m+1}(0)=\mathbf{0} .
$$

Now, (3.5) in particular means that

$$
J_{M} f_{m+1}(0) J_{M}=f_{m+1}(m+1) .
$$

The paraunitary condition in the time domain implies $f_{m+1}^{T}(m+1) f_{m+1}(0)=\mathbf{0}$. Hence, we have

$$
\boldsymbol{f}_{m+1}^{T}(0) \boldsymbol{J}_{M} \boldsymbol{f}_{m+1}(0)=\mathbf{0} .
$$

By Sylvester's rank inequality [30] therefore, we get $\operatorname{rank}\left(f_{m+1}(0)\right)=r \leq M / 2$.

Equation (3.28) implies that the columns of the matrix $\boldsymbol{f}_{m+1}(0)$ are balanced. Hence, it can be shown (Appendix B), that there exists a set of orthonormal balanced vectors $\boldsymbol{x}_{i}, i=1, \cdots, M / 2$ such that if $\boldsymbol{X}^{T}$ is the matrix of size $M / 2 \times M$ whose rows are these vectors, this matrix satisfies the following properties:

1) $\boldsymbol{X}^{T} \boldsymbol{X}=\boldsymbol{I}_{M / 2}$ (from the fact that $\boldsymbol{x}_{i}$ are orthogonal).

2) $\boldsymbol{X}^{T} \boldsymbol{J}_{M} \boldsymbol{X}=0$ (from the fact that $\boldsymbol{x}_{i}$ are balanced).

3) $\boldsymbol{X}^{T} J_{M} f_{m+1}(0)=\mathbf{0}$ (by the construction outlined in Appendix B).

It can be verified that the matrix

$$
\boldsymbol{T}_{m+1}=\left(\begin{array}{c}
\boldsymbol{X}^{T} \\
\boldsymbol{X}^{T} \boldsymbol{J}_{M}
\end{array}\right)\left(\begin{array}{cc}
\boldsymbol{I}_{M / 2} & \mathbf{0} \\
\mathbf{0} & \boldsymbol{J}_{M / 2}
\end{array}\right)
$$

can be written in the form as in (3.24). Moreover, with this choice of the matrix $\boldsymbol{T}_{m+1},(3.25)$ is satisfied. This proves that $\boldsymbol{F}_{m}(z)$ is causal.

Order reduction: Given the fact that $\boldsymbol{F}_{m}(z)$ is causal, and that it satisfies (3.2), we can see that the order of $\boldsymbol{F}_{m}(z)$ is $m$. Thus there is a reduction in order by 1 . Hence, for a system of order $N$, the factorization process is guaranteed to terminate in $N$ steps.

This concludes the proof of Theorem 3 .

The above theorem guarantees the factorization of all linear phase paraunitary systems satisfying (2.1). Such a linear phase filter bank with polyphase matrix of order $N$ can hence be characterized by $2(N)\left({ }_{2}^{M / 2}\right)$ rotation angles.

The degree of a causal rational system is defined as $[10$, sec. 13.8] the minimum number of delays required for its implementation. A structure is said to be minimal if the number of delays used is equal to the degree of the transfer function. For a paraunitary system, we know that $[10$, Theorem 14.7.1] that

$$
\operatorname{deg}[\operatorname{det}[\boldsymbol{E}(z)]]=\operatorname{deg}[\boldsymbol{E}(z)] .
$$

In our case,

$$
\begin{aligned}
& \operatorname{deg}[\operatorname{det}[\boldsymbol{E}(z)]]=\operatorname{deg}\left[\operatorname { d e t } \left[\boldsymbol{S P P} \boldsymbol{T}_{N} \boldsymbol{\Lambda}(z) \boldsymbol{T}_{N-1}\right.\right. \\
& \left.\left.\cdot \boldsymbol{\Lambda}(z) \cdots \boldsymbol{\Lambda}(z) \boldsymbol{T}_{0} \boldsymbol{P}\right]\right]=N M / 2
\end{aligned}
$$

which is equal to the number of delays used. Hence, the factorization is minimal.

\section{Linear Phase Paraunitary Filters with Pairwise Mirror-IMage Frequency RESPONSES FOR EVEN $M$}

In the previous section, we factorized a linear phase paraunitary system into a product of orthogonal building blocks each of which can be implemented with $2\left({ }_{2}^{M / 2}\right)$ rotation angles. These angles can be made the variables in the design process. The number of angles can be become fairly large when the number of channels $M$ increases. It would be useful to cut down the number of optimization variables by structurally imposing some other additional constraints on the filters. One of the constraints that can be imposed is that of pairwise mirror image symmetry in the frequency domain around $\pi / 2$. Such a condition had been imposed on general paraunitary systems in [31]. One way to impose the condition that the filter satisfy the pairwise mirror image condition in the frequency domain is to ensure that the filters are related as

$$
H_{M-1-k}(z)=H_{k}(-z), \quad k=0, \cdots, L-1
$$

where $L=M / 2$. If $M$ is even, in terms of the polyphase matrix of the filters this becomes

$$
\boldsymbol{J}_{M} \boldsymbol{E}(z)=\boldsymbol{E}(z) \boldsymbol{V}_{M} .
$$

As mentioned earlier, the matrix $V_{M}$ is a diagonal matrix of size $M \times M$ with alternate \pm 1 's on the diagonal, starting with +1 . This symmetry condition is in addition to the conditions of linear phase (2.1) and paraunitariness (1.1).

To develop a cascade structure which generates such filters, we will assume that we have a paraunitary matrix $\boldsymbol{E}_{m-1}(z)$ of order $m-1$ satisfying the conditions of paraunitariness (1.1), linear phase (2.1), and pairwise mirrorimage symmetry of frequency responses (4.2). From it, we will show how a paraunitary matrix $\boldsymbol{E}_{m}(z)$ of order $m$ can be obtained satisfying the above three properties. We will do this by post multiplying the given matrix $\boldsymbol{E}_{m-1}(z)$ by a paraunitary matrix $\boldsymbol{R}(z)$ of order one.'

Let

$$
\boldsymbol{E}_{m}(z)=\boldsymbol{E}_{m-1}(z) \boldsymbol{R}(z) .
$$

Clearly, $\boldsymbol{E}_{m}(z)$ is paraunitary. Also,

$$
\boldsymbol{E}_{m-1}(z)=\boldsymbol{E}_{m}(z) \tilde{\boldsymbol{R}}(z) .
$$

Propagating the Linear Phase Property: From the fact that $\boldsymbol{E}_{m-1}(z)$ satisfies the linear phase property, we have

$$
z^{-m} \boldsymbol{D} \boldsymbol{E}_{m}\left(z^{-1}\right) \tilde{\boldsymbol{R}}\left(z^{-1}\right) \boldsymbol{J}_{M}=\boldsymbol{E}_{m}(z) \tilde{\boldsymbol{R}}(z)
$$

${ }^{\mathrm{I}}$ This derivation could also be made by premultiplying an existing matrix by an extra block. This was the approach followed in Section III, because it simplifies the proof of Theorem 3 to some extent. In proving the results of this section, the postmultiplication strategy will lead to slightly simpler derivations. The reader must note that preference for one strategy over the other has been dictated purely by simplicity of presentation. 
i.e.,

$$
z^{-m} \boldsymbol{D} \boldsymbol{E}_{m}\left(z^{-1}\right) \tilde{\boldsymbol{R}}\left(z^{-1}\right) \boldsymbol{J}_{M} \boldsymbol{R}(z)=\boldsymbol{E}_{m}(z)
$$

Hence for $\boldsymbol{E}_{m}(z)$ to satisfy the linear phase property, $\boldsymbol{R}(z)$ should satisfy

$$
\tilde{\boldsymbol{R}}\left(z^{-1}\right) \boldsymbol{J}_{M} \boldsymbol{R}(z)=z^{-1} \boldsymbol{J}_{M}
$$

It can be verified that if $\boldsymbol{R}(z)=\boldsymbol{\Lambda}(z) \boldsymbol{P T P}$ with the matrices $\boldsymbol{\Lambda}(z), \boldsymbol{P}$ and $\boldsymbol{T}$ as in the previous section, $\boldsymbol{R}(z)$ sat isfies (4.7).

Propagating the Pairwise Mirror-Image Property in the Frequency Domain: Assuming that (4.2) holds for $\boldsymbol{E}_{m-1}(z)$, and using (4.4) we get

$$
\boldsymbol{J}_{M} \boldsymbol{E}_{m}(z) \tilde{\boldsymbol{R}}(z)=\boldsymbol{E}_{m}(z) \tilde{\boldsymbol{R}}(z) \boldsymbol{V}_{M}
$$

i.e.,

$$
\boldsymbol{J}_{M} \boldsymbol{E}_{m}(z)=\boldsymbol{E}_{m}(z) \tilde{\boldsymbol{R}}(z) \boldsymbol{V}_{M} \boldsymbol{R}(z) .
$$

Hence, $\boldsymbol{R}(z)$ should satisfy the property

$$
\tilde{\boldsymbol{R}}(z) \boldsymbol{V}_{M} \boldsymbol{R}(z)=\boldsymbol{V}_{M} .
$$

We now have two cases:

Case 1: $M / 2$ is even: In this case, $V_{M}=\left(\begin{array}{ll}V_{M} / 2 & \mathbf{V}_{M / 2}\end{array}\right)$. Substituting this, and the fact that $\boldsymbol{R}(z)=\boldsymbol{\Lambda}(z) \boldsymbol{P T P}$ with $\boldsymbol{T}=\left(\begin{array}{cc}{ }_{C}^{A} & { }_{A}^{C}\end{array}\right)$, in (4.10) and simplifying, we get

$$
\begin{gathered}
\left(\begin{array}{ll}
\boldsymbol{A}^{T} & \boldsymbol{C}^{T} \\
\boldsymbol{C}^{T} & \boldsymbol{A}^{T}
\end{array}\right)\left(\begin{array}{cc}
\boldsymbol{V}_{M / 2} & \mathbf{0} \\
\mathbf{0} & -\boldsymbol{V}_{M / 2}
\end{array}\right)\left(\begin{array}{ll}
\boldsymbol{A} & \boldsymbol{C} \\
\boldsymbol{C} & \boldsymbol{A}
\end{array}\right) \\
=\left(\begin{array}{cc}
\boldsymbol{V}_{M / 2} & \mathbf{0} \\
\mathbf{0} & -\boldsymbol{V}_{M / 2}
\end{array}\right) .
\end{gathered}
$$

Using a factorization for $\boldsymbol{T}$ similar to (3.12) and simplifying, we get

$$
\left(\begin{array}{cc}
\mathbf{0} & \boldsymbol{U}^{T} \boldsymbol{V}_{M / 2} \boldsymbol{W} \\
\boldsymbol{W}^{T} \boldsymbol{V}_{M / 2} \boldsymbol{U} & \mathbf{0}
\end{array}\right)=\left(\begin{array}{cc}
\mathbf{0} & \boldsymbol{V}_{M / 2} \\
\boldsymbol{V}_{M / 2} & \mathbf{0}
\end{array}\right) .
$$

The above equation is satisfied if $U$ is taken to be an arbitrary orthogonal matrix of size $M / 2 \times M / 2$, and the matrix $\boldsymbol{W}$ is chosen as

$$
\boldsymbol{W}=\boldsymbol{V}_{M / 2} \boldsymbol{U} \boldsymbol{V}_{M / 2}
$$

Hence, in this case, we have $\left(\begin{array}{l}M / 2 \\ 2\end{array}\right)$ degrees of freedom to optimize per stage.

Case 2: $M / 2$ is odd: In this case, $V_{M}=$ $\left(\begin{array}{ll}V_{M / 2} & 0 \\ 0 & -V_{M / 2}\end{array}\right)$, unlike the case where $M / 2$ is even. However, if we use the relation $\boldsymbol{R}(z)=\boldsymbol{\Lambda}(z) \boldsymbol{P T P}$ with $\boldsymbol{T}=$ $\left(\begin{array}{ll}A & C \\ C & A\end{array}\right)$, and perform the simplifications as before, we get (4.12) once again, proving that there are $\left(\begin{array}{l}M / 2 \\ 2\end{array}\right)$ degrees of freedom to be optimized in this case also.

Thus, all three properties have been satisfied.

Initialization: It only remains to find a degree zero paraunitary matrix $\boldsymbol{E}_{0}(z)$ (i.e., a constant orthogonal matrix $S$ ), which will initialize the above process. From the discussion in Section III it can be verified that the matrix $S$ satisfies the linear phase property $\left(D S J_{M}=S\right)$, if it is of the form

$$
\boldsymbol{S}=(1 / \sqrt{2})\left(\begin{array}{cc}
\boldsymbol{S}_{0} & \mathbf{0} \\
\mathbf{0} & \boldsymbol{S}_{1}
\end{array}\right)\left(\begin{array}{rr}
\boldsymbol{I}_{M / 2} & \boldsymbol{J}_{M / 2} \\
\boldsymbol{I}_{M / 2} & -\boldsymbol{J}_{M / 2}
\end{array}\right) \boldsymbol{Q}
$$

where $Q$ is a symmetric permutation matrix. This is because $Q J_{M} Q=J_{M}$ for any such permutation matrix. Let $Q$ be so chosen that $Q V_{M} Q=D$, where $D=$ $\left(_{0}^{I_{M / 2}} \quad{ }_{-}^{0} I_{M / 2}\right)$. Now, let $S^{\prime}=S \boldsymbol{Q}$. For the matrix $\boldsymbol{S}$ to satisfy the pairwise mirror-image property $\left(J_{M} S=S V_{M}\right)$, it can be verified that the matrix $S^{\prime}$ should satisfy $S^{\prime} D S^{\prime T}$ $=J_{M}$. Substituting the forms of various matrices and simplifying, we get

$$
\left(\begin{array}{cc}
\mathbf{0} & \boldsymbol{S}_{0} \boldsymbol{S}_{1}^{T} \\
\boldsymbol{S}_{1} \boldsymbol{S}_{0}^{T} & \mathbf{0}
\end{array}\right)=\left(\begin{array}{cc}
\mathbf{0} & \boldsymbol{J}_{M / 2} \\
\boldsymbol{J}_{M / 2} & \mathbf{0}
\end{array}\right)
$$

This equation can be satisfied by letting $S_{0}$ be an arbitrary orthogonal matrix, and choosing $S_{1}=J_{M / 2} S_{0}$. Thus the matrix $S$ can be realized with $\left({ }_{2}^{M / 2}\right)$ rotations [29].

The foregoing discussion can be summarized in the following theorem:

Theorem 4: A linear phase paraunitary matrix satisfying (2.1) whose filters satisfy the additional pairwise mirror-image property in the frequency domain (4.2) can be realized as

$$
\boldsymbol{E}(z)=\boldsymbol{S \Lambda}(z) \boldsymbol{P} \boldsymbol{T}_{0} \boldsymbol{\Lambda}(z) \cdots \boldsymbol{T}_{N} \boldsymbol{P}
$$

where

$$
\begin{aligned}
\boldsymbol{S}= & (1 / \sqrt{2})\left(\begin{array}{cc}
\boldsymbol{S}_{0} & \mathbf{0} \\
\mathbf{0} & \boldsymbol{J}_{M / 2} \boldsymbol{S}_{0}
\end{array}\right)\left(\begin{array}{cc}
\boldsymbol{I} & \boldsymbol{J}_{M / 2} \\
\boldsymbol{I} & -\boldsymbol{J}_{M / 2}
\end{array}\right) \boldsymbol{Q}, \\
\boldsymbol{T}_{i}= & \left(\begin{array}{cc}
\boldsymbol{I}_{M / 2} & \boldsymbol{I}_{M / 2} \\
\boldsymbol{I}_{M / 2} & -\boldsymbol{I}_{M / 2}
\end{array}\right)\left(\begin{array}{cc}
\boldsymbol{V}_{M / 2} \boldsymbol{U}_{i} \boldsymbol{V}_{M / 2} & \mathbf{0} \\
\mathbf{0} & \boldsymbol{U}_{i}
\end{array}\right) \\
& \cdot\left(\begin{array}{rr}
\boldsymbol{I}_{M / 2} & \boldsymbol{I}_{M / 2} \\
\boldsymbol{I}_{M / 2} & -\boldsymbol{I}_{M / 2}
\end{array}\right)
\end{aligned}
$$

and the matrix $\boldsymbol{P}$ is as in (3.10).

The fact that the structure continues to be minimal is easily verified, though we have not shown it to be complete.

Fig. 5 shows an example of a 8-channel system (Design ex. 1) where a 4-stage lattice was used. The filters are linear phase, paraunitary, and satisfy the pairwise mirrorimage symmetry in the frequency domain. The impulse response coefficients of the 8 -channel system have been tabulated in Table I. Fig. 6 shows a similar example for the 4-channel case (Design ex. 2). The impulse response coefficients have been tabulated in Table II.

The coding gain [18] is often used as a figure of merit to judge the performance of various subband coding schemes. It is defined as the ratio of the reconstruction error variance of a PCM system to the reconstruction error variance of the subband coding system. It was verified through examples that the imposition of linear phase property did not lead to significant coding gain reduction. 


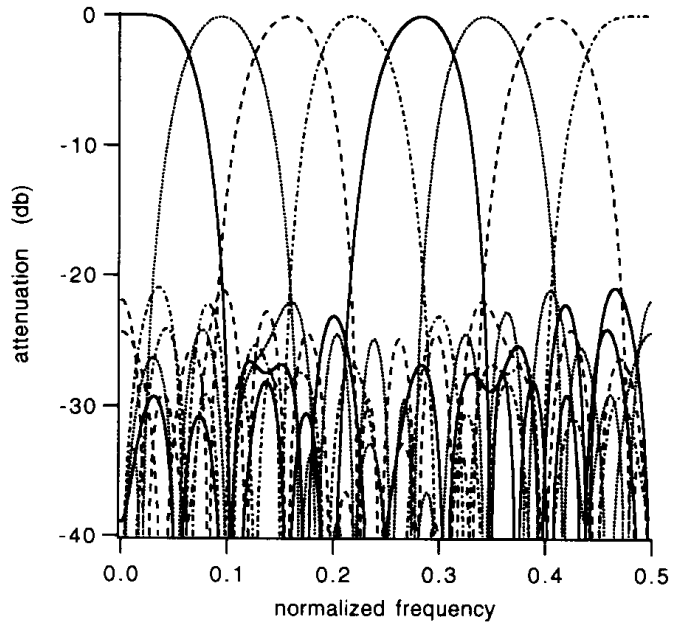

Fig. 5. (Design ex. 1) Magnitude responses of a 8-channel linear phase paraunitary filter bank. The filters have length 32 each.

TABLE I

(Design ex. 1) Filter Coefficients of a 8-Channel linear Phase Paraunitary Filter Bank. [Only the CoefFicients $h_{i}(0)$ Through $h_{i}(15)$ are Tablilated for the First Four Filters. Linear Phase IMPLIES $h_{i}(n)=h_{i}(31-n)$. AND MIRROR-IMAGE SYMMETRY IMPLIES $\left.H_{i}(-z)=H_{7-i}(z)\right]$. THE FILTER LENGTH IS 32

\begin{tabular}{|c|c|c|c|}
\hline $\mathrm{H}_{0}(\mathrm{z})$ & $\mathrm{H}_{1}(\mathrm{z})$ & $\mathrm{H}_{2}(\mathrm{z})$ & $\mathrm{H}_{3}(\mathrm{z})$ \\
\hline 2.70636 & 9692058 & $69506430012 \mathrm{D}$ & $1960492098433 \mathrm{D}-102$ \\
\hline-1.2136 & & & \\
\hline $\begin{array}{l}1.216 \\
.093\end{array}$ & $55274487 \mathrm{D}-03$ & $000-02$ & $-4.0347878743950 \mathrm{D}$ \\
\hline $\begin{array}{l}-1.0939 \\
-34006\end{array}$ & 494340 & 600 & $2928325761109 \mathrm{D}-02$ \\
\hline $\begin{array}{l}3.9008362624561 \mathrm{D}-02 \\
17129046826691 \mathrm{D}-02\end{array}$ & 1.410 & 6ID-02 & D \\
\hline $6.2580468711733 \mathrm{D}-02$ & $\begin{array}{c}-1.1144664575007 \mathrm{D}-02 \\
409752480522\end{array}$ & $\begin{array}{l}3.4018237002281 \mathrm{D}-03 \\
9\end{array}$ & -4.94448345 \\
\hline $.5970643737541 \mathrm{D}-02$ & $\begin{array}{l}4.09753248505240-02 \\
012106010509825\end{array}$ & $8.6349676608556 \mathrm{D}-03$ & $\begin{array}{l}-3.7266345907583 \mathrm{D}-02 \\
8\end{array}$ \\
\hline $085817350597 \mathrm{D}-02$ & 0.12469607197029 & $-7.7589420570820 \mathrm{D}-02$ & $\begin{array}{l}8.4900080405164 \mathrm{DD}-122 \\
01186664669675\end{array}$ \\
\hline $124808276196 \mathrm{D}-02$ & 0.15395817207336 & -0.22772066314211 & 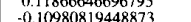 \\
\hline $1.0067593331610 \mathrm{D}-01$ & $1.6326854841075 \mathrm{D}-02$ & -0.19287393785391 & 026124185969832 \\
\hline 0.17114723136171 & -0.14257162890702 & 0.10867205234840 & $-1.3044768601487 \mathrm{D}-1) 3$ \\
\hline 0.24072321382144 & -0.34043725682195 & 9903907 & 0.37437930463834 \\
\hline 0.32076121985196 & -0.41375436862609 & 4416569 & 0.12094472643289 \\
\hline 0.355025 & -0.33699605405521 & $-3.4833482443945 \mathrm{D}-02$ & -0.389520446170 \\
\hline & -0.14275844384806 & -0.40959004960062 & -0.292274603555 \\
\hline
\end{tabular}

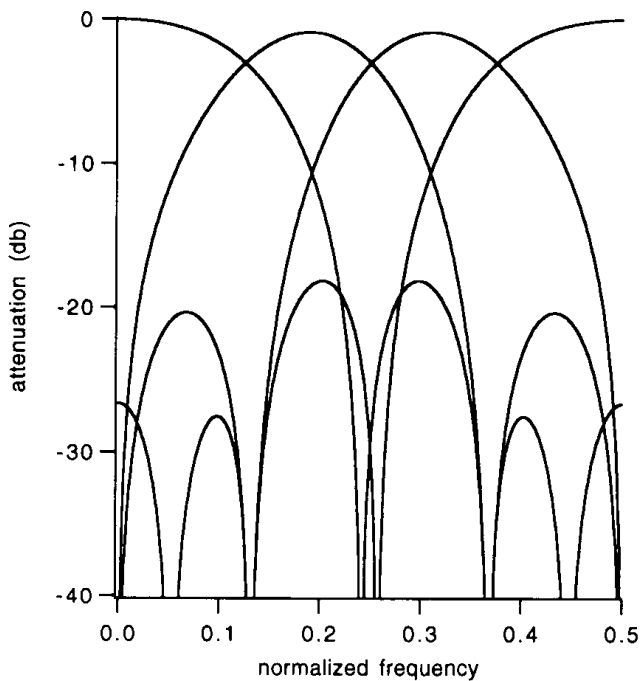

Fig. 6. (Design ex. 2) Magnitude responses of a 4-channel linear phase paraunitary filter bank. The filters have length 8 each.
TABLE II

(Design ex. 2) Filter Coefficients of a 4-Channel Linear Phase Paraunitary Filter Bank. Note that $H_{3}(z)=H_{0}(-z)$ and $H_{2}(z)=$ $H_{1}(-z)$. ThE FILTER LeNGTH IS 8

\begin{tabular}{l|l}
\hline coefficients of $H_{0}(z)$ & coefficients of $H_{1}(z)$ \\
\hline$h_{0}(0)=h_{0}(7)=-0.091584806958951$ & $h_{1}(0)=-h_{1}(7)=-0.13357390156568$ \\
$h_{0}(1)=h_{0}(6)=0.13357390156568$ & $h_{1}(1)=-h_{1}(6)=0.091584806958951$ \\
$h_{0}(2)=h_{0}(5)=0.38923341521735$ & $h_{1}(2)=-h_{1}(5)=0.56768614376856$ \\
$h_{0}(3)=h_{0}(4)=0.56768614376856$ & $h_{1}(3)=-h_{1}(4)=0.38923341521735$ \\
\hline
\end{tabular}

As an example, using bandpass speech as input, imposition of the linear phase property reduced the coding gain from $6.2 \mathrm{db}$ to $5.2 \mathrm{db}$.

\section{Linear Phase Paraunitary Filters for Odd $M$}

While the existence of linear phase paraunitary filter banks had been indicated in [26] for an even number of channels $M$, for an odd number of channels, the existence of nondegenerate filter banks has not been shown so far. In this section, we shall synthesize linear phase paraunitary filter banks for an odd number of channels. There are two ways to design such systems. One way is to develop a cascade structure as we did in the previous sections. The second way is to obtain linear phase systems for a certain odd $M$ by suitably combining linear phase systems of size $(M-1) / 2$ and $(M+1) / 2$, while maintaining the paraunitary property. We will consider both of these approaches in this section.

\section{A. A Cascade Based Approach}

In this subsection we proceed as we did in Section III, i.e., first design a set of filters which satisfy the property that the filters are pairwise flipped versions of each other in the time domain, and then suitably combine these to get a linear phase system.

Fact 4.1: Consider a polyphase matrix of size $M \times M$, $M$ odd, which is obtained as the following product:

$$
\boldsymbol{F}(z)=\boldsymbol{P} \boldsymbol{T}_{N} \boldsymbol{\Lambda}(z) \boldsymbol{T}_{N-1} \boldsymbol{\Lambda}(z) \cdots \boldsymbol{\Lambda}(z) \boldsymbol{T}_{0} \boldsymbol{P}
$$

where

$$
\boldsymbol{P}=\left(\begin{array}{cc}
I_{(M+1) / 2} & \mathbf{0} \\
\mathbf{0} & \boldsymbol{J}_{(M-1) / 2}
\end{array}\right)
$$

$\boldsymbol{T}_{i}$ are orthogonal matrices of the form

$$
\boldsymbol{T}_{i}=\left(\begin{array}{lll}
\boldsymbol{A}_{i} & \mathbf{0} & \boldsymbol{C}_{i} \\
\mathbf{0}^{T} & 1 & \mathbf{0}^{T} \\
\boldsymbol{C}_{i} & \mathbf{0} & \boldsymbol{A}_{i}
\end{array}\right)
$$

and

$$
\boldsymbol{\Lambda}(z)=\left(\begin{array}{cc}
\boldsymbol{I}_{(M+1) / 2} & \mathbf{0} \\
\mathbf{0} & z^{-1} \boldsymbol{I}_{(M-1) / 2}
\end{array}\right)
$$

Then this structure generates a paraunitary filter bank in which $H_{k}(z)$ is the time-reversed version of $H_{M-1-k}(z)$. 
Proof: Since all matrices in the product are individually paraunitary, the product $\boldsymbol{F}(z)$ is also paraunitary. Now to prove that the filters are pairwise flipped versions of one another, we need to show that the matrix $\boldsymbol{F}(z)$ satisfies the condition

$$
\left(\begin{array}{ccc}
z^{-N} \boldsymbol{I}_{(M-1) / 2} & \mathbf{0} & \mathbf{0} \\
\mathbf{0}^{T} & 1 & \mathbf{0}^{T} \\
\mathbf{0} & \mathbf{0} & z^{-N} \boldsymbol{I}_{(M-1) / 2}
\end{array}\right) \boldsymbol{J}_{M} \boldsymbol{F}\left(z^{-N}\right) \boldsymbol{J}_{M}=\boldsymbol{F}(z)
$$

where $N$ is the order of the polyphase matrix $F(z)$. In particular, by our construction, the middle filter will be just $H_{(M+1) / 2}^{\prime}(z)=z^{-(M+1) / 2}$. Hence, it is the flipped version of itself. Substituting the forms of various matrices, we can verify that $(5.5)$ indeed holds.

Now suppose we are given a paraunitary matrix $F(z)$ satisfying (5.5) and whose order $N$ is even (as required by Lemma 1), we can obtain a matrix $\boldsymbol{E}(z)$ from it which corresponds to a set of linear phase paraunitary filters. Since the middle filter is just $H_{(M+1) / 2}^{\prime}(z)=z^{-(M+1) / 2}$, we first multiply this filter by an appropriate delay $z^{-N / 2}$. This can be done by premultiplying the matrix $F(z)$ by the diagonal matrix $\boldsymbol{\Lambda}^{\prime}(z)=\operatorname{diag}\left[\begin{array}{ll}1 & 1\end{array}\right.$ $\cdots z^{-N / 2} \cdots 1$ 1] to get $\boldsymbol{F}^{\prime}(z)=\boldsymbol{\Lambda}^{\prime}(z) \boldsymbol{F}(z)$. The matrix $\boldsymbol{F}^{\prime}(z)$ hence satisfies the equation

$$
z^{-N} \boldsymbol{J}_{M} \boldsymbol{F}^{\prime}\left(z^{-1}\right) \boldsymbol{J}_{M}=\boldsymbol{F}^{\prime}(z)
$$

Now let $\boldsymbol{E}(z)=\boldsymbol{S} \boldsymbol{F}^{\prime}(z)$ where $\boldsymbol{S}$ is an orthogonal matrix. Clearly, with this construction, the matrix $\boldsymbol{E}(z)$ is
Theorem 5: A linear phase paraunitary matrix with an odd number of channels can be realized as

$$
\boldsymbol{E}(z)=\boldsymbol{S} \boldsymbol{\Lambda}^{\prime}(z) \boldsymbol{P} \boldsymbol{T}_{N} \boldsymbol{\Lambda}(z) \boldsymbol{T}_{N-1} \boldsymbol{\Lambda}(z) \cdots \boldsymbol{T}_{0} \boldsymbol{P}
$$

where $\boldsymbol{P}$ is as in (5.2), $\boldsymbol{T}_{i}$ is as in (5.3) and $\boldsymbol{\Lambda}(z)$ is as in (5.4).

The fact that the structure is minimal can be verified as at the end of Section III.

\section{B. Matrix Interleaving and Linear Phase Filters}

In this subsection, we will consider the problem of obtaining a larger linear phase paraunitary system given smaller linear phase paraunitary systems. Let $M$, the number of channels be odd. Let $L=(M-1) / 2$. Let $\boldsymbol{G}(z)$ and $\boldsymbol{F}(z)$ be two linear phase paraunitary matrices of sizes $(L+1) \times(L+1)$ and $L \times L$ respectively, and of order $N$ each. In particular, let us write them as

$$
\boldsymbol{G}(z)=\left(\begin{array}{lllll}
\boldsymbol{g}_{0}(z) & \boldsymbol{g}_{1}(z) & \cdots & \boldsymbol{g}_{L-1}(z) & \boldsymbol{g}_{L}(z) \\
g_{0}^{\prime}(z) & g_{1}^{\prime}(z) & \cdots & g_{L-1}^{\prime}(z) & g_{L}^{\prime}(z)
\end{array}\right)
$$

and

$$
\boldsymbol{F}(z)=\left(f_{0}(z) f_{1}(z) \cdots f_{L-2}(z) \quad f_{L-1}(z)\right) .
$$

In (5.9), the vectors $g_{i}(z)$ are of size $L$, and represent the columns of the matrix $\boldsymbol{G}(z)$, except for the last element in each column, which has been written separately as $g_{i}^{\prime}(z)$. In (5.10), the vectors $f_{i}(z)$ are also of size $L$, and are simply the columns of the matrix $F(z)$. Hence note that vectors $g_{i}(z)$ and $f_{i}(z)$ are all of size $L$ each. Now, construct the matrix $\boldsymbol{E}(z)$ of size $M \times M$, which is as follows:

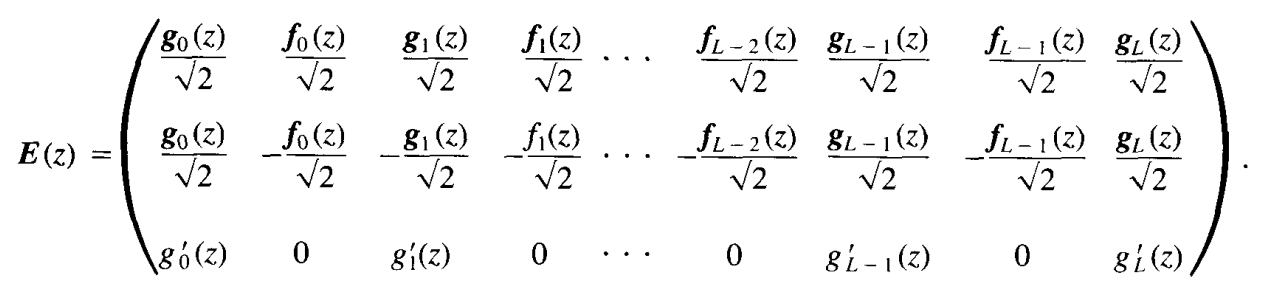

also paraunitary. For the matrix $\boldsymbol{E}(z)$ to satisfy the linear phase property (2.1), it can be verified that it is both necessary and sufficient that $S$ be of the form

$$
\begin{aligned}
\boldsymbol{S}= & (1 / \sqrt{ } 2)\left(\begin{array}{cc}
\boldsymbol{U}_{(M+1) / 2} & \mathbf{0} \\
\mathbf{0} & \boldsymbol{W}_{(M-1) / 2}
\end{array}\right) \\
& \cdot\left(\begin{array}{ccc}
\boldsymbol{I}_{(M-1) / 2} & \mathbf{0}_{c} & \boldsymbol{J}_{(M-1) / 2} \\
\boldsymbol{0}_{c}^{T} & 1 & \boldsymbol{0}_{c}^{T} \\
\boldsymbol{J}_{(M-1) / 2} & \mathbf{0}_{c} & -\boldsymbol{I}_{(M-1) / 2}
\end{array}\right)
\end{aligned}
$$

where $\boldsymbol{U}_{(M+1) / 2}$ and $\boldsymbol{W}_{(M-1) / 2}$ are arbitrary orthogonal matrices of the sizes indicated.

The above discussions can be summarized in the following theorem:
Note that the filters corresponding to this polyphase matrix are formed simply by interleaving in a particular manner the impulse response coefficients of the filters in the smaller systems $\boldsymbol{G}(z)$ and $\boldsymbol{F}(z)$.

Lemma 2: The matrix $\boldsymbol{E}(z)$ of size $M \times M$ in (5.11) is a linear phase paraunitary matrix of order $N$.

Proof: The fact that $\boldsymbol{E}(z)$ is paraunitary is clear from the construction. It only remains to prove the linear phase property. Because the matrices $\boldsymbol{G}(z)$ and $\boldsymbol{F}(z)$ are linear phase, we have the following relations

$$
\begin{aligned}
& \boldsymbol{g}_{i}(z)= \pm z^{-N} \boldsymbol{g}_{L-i}\left(z^{-1}\right), \\
& \boldsymbol{f}_{i}(z)= \pm z^{-N} \boldsymbol{f}_{L-i-1}\left(z^{-1}\right)
\end{aligned}
$$

and

$$
g_{i}^{\prime}(z)= \pm z^{-N} g_{L-i}^{\prime}\left(z^{-1}\right) .
$$




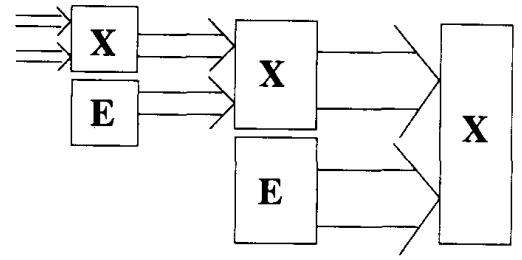

$\mathbf{E}=$ structure giving linear-phase paraunitary filters for even $\mathrm{M}$ $\mathbf{X}=$ the interleaving mechanism

Fig. 7. Obtaining linear phase paraunitary filters by interleaving smaller systems.

Let $\boldsymbol{e}_{i}(z)$ denote the columns of the matrix $\boldsymbol{E}(z)$. Then, it can be seen from the construction of the matrix $\boldsymbol{E}(z)$ that the columns satisfy the condition

$$
e_{i}(z)= \pm z^{-N} e_{M-1-i}\left(z^{-1}\right)
$$

This is sufficient to prove that $\boldsymbol{E}(z)$ has linear phase filters.

Lemma 2 gives us a way to synthesize larger paraunitary systems from smaller ones. Thus, one can obtain a $M$ channel linear phase paraunitary filter bank by using a schematic as shown in Fig. 7. Here, Lemma 2 is repeatedly used to synthesize the odd component on each level.

\section{VI. $M$-Band Orthonormal Wavelets}

The wavelet transform [32]-[34], [13] is a representation of a signal in terms of a set of basis functions which are obtained by dyadic dilations and shifts of a single function called the wavelet function. It provides a description of a signal on various levels of resolution or scale. The wavelet transform has of late, found several applications in signal and image processing [34], [35]. One way of constructing the wavelet functions that generate a basis [32] is by using a two-channel quadraturemirror filter bank is an infinite tree. This idea of wavelets (henceforth referred to as dyadic wavelets) has recently been extended to the more general case of $M$-band wavelets [19], [20], [36]. It has been shown therein that a square integrable function $f(t)$ can be represented in terms of the dilates and translates of $M-1$ functions $\psi_{i}(t)$, which are called the $M$-band wavelets. As in the case of dyadic wavelets, it has been shown [20] that $M$-band wavelets can be obtained by using a $M$-channel filter bank system in a infinite recursive tree-structure as shown in Fig. 8. $M$-band wavelets often provide a more compact representation of signals, and are therefore useful in several applications [21].

It can be shown [20], that for the wavelet basis to be orthonormal, a necessary condition is that the $M$-channel filter bank used in Fig. 8 should be paraunitary. The theory developed in the previous sections allows us to design symmetric and antisymmetric wavelets that are also orthonormal. This can be done simply by using the structure developed in Section III to generate the $M$-channel system

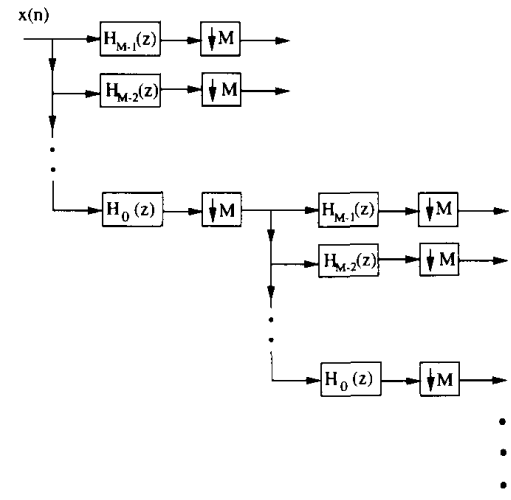

Fig. 8. A tree-structure using $M$-channel filter bank.

on each level of the tree. Consider the quantity

$$
\begin{aligned}
\Psi_{i}(\omega)= & (1 / \sqrt{M}) H_{i}\left(e^{j \omega / M}\right) \\
& \cdot \lim _{K \rightarrow \infty} \prod_{k=2}^{K}(1 / \sqrt{M}) H_{0}\left(e^{j \omega(M)^{-k}}\right)
\end{aligned}
$$

where $H_{0}\left(e^{j \omega}\right)$ is a rational (in fact FIR) filter. This converges pointwise for all $\omega$ as long as $\left|H_{0}\left(e^{j \omega}\right)\right| \leq \sqrt{M}$ and $H_{0}\left(e^{j 0}\right)=\sqrt{M}$. For the linear phase paraunitary system developed in Section III, the filters can be written as $H_{i}\left(e^{j \omega}\right)=e^{-j(N-1) \omega / 2} H_{i R}(\omega)$, where $H_{i R}(\omega)$ is the real part of $H_{i}\left(e^{j \omega}\right)$, and we have

$$
\begin{gathered}
\Psi_{i}(\omega)=e^{-j(N-1) \omega\left(M^{-1}+M^{-2}+\cdots\right) / 2}(1 / \sqrt{M}) H_{i R}(\omega) \\
\cdot \lim _{K \rightarrow \infty} \prod_{k=2}^{K}(1 / \sqrt{M}) H_{0 R}\left(\omega(M)^{-k}\right)
\end{gathered}
$$

which becomes

$$
\begin{gathered}
\Psi_{i}(\omega)=e^{-j(N-1) \omega /(2(M-1))} \lim _{K \rightarrow \infty}(1 / \sqrt{M}) H_{i R}(\omega) \\
\cdot \prod_{k=2}^{K}(1 / \sqrt{M}) H_{0 R}\left(\omega(M)^{-k}\right)
\end{gathered}
$$

This is the Fourier Transform of an $M$-band wavelet function and has linear phase.

Convergence, Orthonormality and Regularity [32]: The RHS in (6.1) always converges pointwise as long as $\left|H_{0}\left(e^{j \omega}\right)\right| \leq \sqrt{M}$, and $H_{0}\left(e^{j 0}\right)=\sqrt{M}$. In the paraunitary case, if the filter $H_{0}\left(e^{j \omega}\right)$ has no zeros in the range $[-\pi / M, \pi / M]$, then convergence is also in the $L_{2}$ sense, and the resulting continuous time functions form an orthonormal basis for $L_{2}$. The orthonormal family is $M^{k / 2} \Psi_{i}\left(M^{k} t-l\right), i=1,2,3$. These facts can be derived by extending the two-channel results of [37], [39].

Fig. 9 shows an example of 4-band orthonormal, linear phase wavelets and their associated scaling function. For this example, the lattice developed in Section IV was used, and the filters were of length 80 each. $H_{0}\left(e^{j \omega}\right)$ was chosen such that it did not have any zero in the range $[-\pi / M, \pi / M]$. Furthermore, in the example, $H_{0}\left(e^{j 0}\right)=$ 

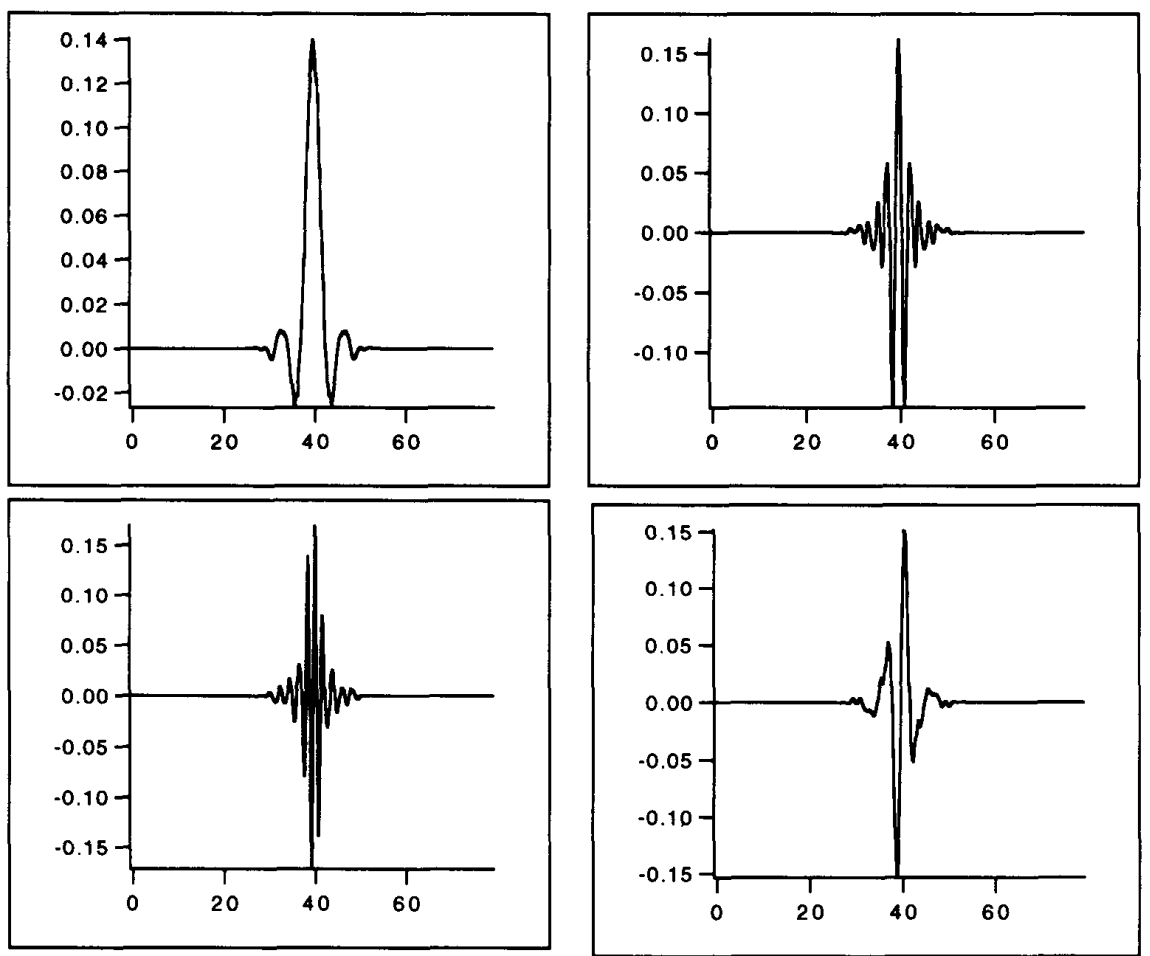

Fig. 9. 4-band linear-phase orthonormal wavelets.

$\sqrt{M}$ has been verified because $\Sigma h_{0}(n)=\sqrt{M}$. Together with paraunitariness, this implies that the continuous time wavelet basis is defined in [20], [36], [38] would be orthonormal. ([37, Theorem 2] can be extended for $M \geq$ 2.) Therefore by power complementarity of the paraunitary filter bank, $H_{k}\left(e^{j 0}\right)=0, k=1,2,3$. By Theorem 3 in [20], this implies that the filter $H_{0}\left(e^{j \omega}\right)$ has at least one zero at the aliasing frequencies $2 \pi k / M, k=1, \cdots, 3$. This means that the continuous time wavelets have at least one vanishing moment [20], [38].

The condition that the continuous time wavelets have at least one vanishing moment can be written directly in terms of the lattice developed in Section III, as was done for the general lattice in [20]. Now, the filters can be written in terms of the polyphase matrix $\boldsymbol{E}(z)$ as

$$
\left(\begin{array}{c}
H_{0}(z) \\
H_{1}(z) \\
\vdots \\
H_{M-1}(z)
\end{array}\right)=\boldsymbol{E}\left(z^{M}\right)\left(\begin{array}{c}
1 \\
z^{-1} \\
\vdots \\
z^{-M+1}
\end{array}\right)
$$

At $\omega=0$, i.e., $z=1$, we need,

$$
\boldsymbol{E}(1)\left(\begin{array}{c}
1 \\
1 \\
\vdots \\
1
\end{array}\right)=\left(\begin{array}{c}
\sqrt{M} \\
0 \\
\vdots \\
0
\end{array}\right)
$$

Substituting the form of the linear phase paraunitary lattice from Section III and noting that $\Lambda(1)=I$, we have

$$
\boldsymbol{S P T}_{N} \boldsymbol{T}_{N-1} \cdots \boldsymbol{T}_{0} \boldsymbol{P}\left(\begin{array}{c}
1 \\
1 \\
\vdots \\
1
\end{array}\right)=\left(\begin{array}{c}
\sqrt{M} \\
0 \\
\vdots \\
0
\end{array}\right) .
$$

Now, with $T_{i}$ having the form as in (3.10), the product $T$ $=\Pi_{i=0}^{n} T_{i}$ also has the form

$$
\boldsymbol{T}=\left(\begin{array}{ll}
\boldsymbol{A} & \boldsymbol{C} \\
\boldsymbol{C} & \boldsymbol{A}
\end{array}\right) .
$$

Similarly, after substituting for the form of $S$ from (3.13), condition (6.6) simplifies to

$$
(\sqrt{2})\left(\begin{array}{cc}
S_{0} & 0 \\
0 & S_{1}
\end{array}\right)\left(\begin{array}{c}
A+C \\
0
\end{array}\right)\left(\begin{array}{c}
1 \\
1 \\
\vdots \\
1
\end{array}\right)=\left(\begin{array}{c}
\sqrt{M} \\
0 \\
\vdots \\
0
\end{array}\right) \text {. }
$$

As expected, this reduces to the set of $M / 2$ conditions

$$
S_{0}(A+C)\left(\begin{array}{c}
1 \\
1 \\
\vdots \\
1
\end{array}\right)=\left(\begin{array}{c}
\sqrt{M / 2} \\
0 \\
\vdots \\
0
\end{array}\right)
$$

where the column vectors are now of size $(M / 2)$. 


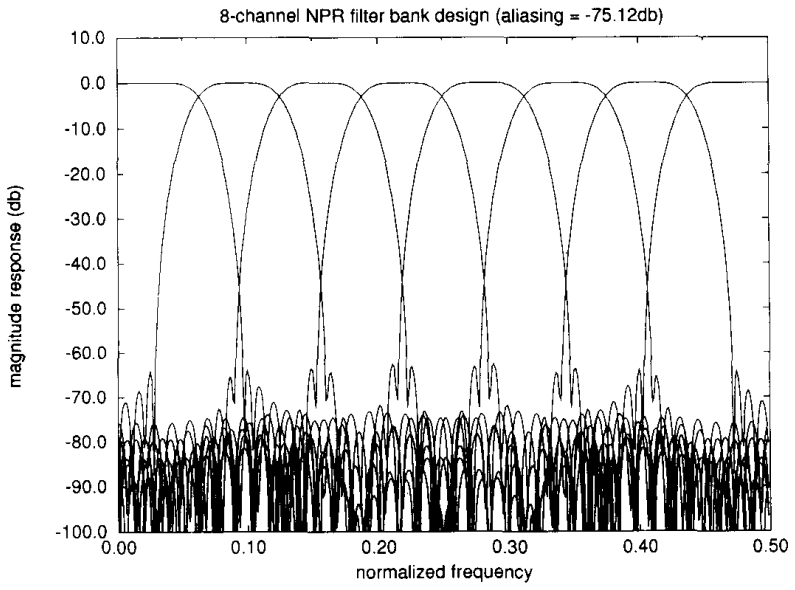

Fig. 10. (Design ex. 3) 8-channel NPR filter bank design (aliasing = $-75.12 \mathrm{db})$.

Recall that $\boldsymbol{S}_{0}$ can be chosen to be an arbitrary orthogonal matrix for the factorization in Section III. If we further wish to impose the condition (6.5), then we can exploit this freedom in the choice of $\boldsymbol{S}_{0}$, and choose it so as to satisfy (6.9). It can be verified that the vector postmultiplying the matrix $S_{0}$ in 6.9 has norm $\sqrt{M / 2}$. Given any vector of norm $\sqrt{M / 2}$, there exists a Householder matrix $I-2 u u^{\dagger}$ which turns the vector into $\left(\begin{array}{c}\sqrt{M / 2} \\ 0 \\ \vdots \\ 0\end{array}\right)[30]$, [10, pp. 751]. So there always exists an $S_{0}$ satisfying (6.9).

\section{Near Perfect Reconstruction Linear Phase DESIGN}

The theory developed in the previous sections approaches the problem of designing linear phase paraunitary systems via structurally imposing the constraints on the filter bank. This results in factorizations of the polyphase matrix. Another approach to designing these filter banks is to formulate the problem as a constrained optimization problem. While the resulting systems do not have perfect reconstruction, they are very close to being perfect reconstruction systems. In this section, we present a design method to obtain the so-called near-perfect-reconstruction (NPR) linear-phase filter banks. The NPR filter banks have some small amplitude distortion and aliasing, however, as we will see, they have high stop-band attenuation. This makes them useful in applications where small distortions can be tolerated. Let $H_{k}(z)$ and $F_{k}(z)$ be the analysis and synthesis linear-phase filters (lengths $L$ ) of a $M$-channel linear-phase filter bank, respectively. The synthesis filters are chosen to be $F_{k}(z)=z^{(L-1)} H_{k}\left(z^{-1}\right)$
$=J_{k} H_{k}(z)$, where $J_{k}$ is 1 (or -1 ) for symmetric (or antisymmetric) analysis filters. The reconstructed output $\hat{X}(z)$ is

$$
\hat{X}(z)=\frac{1}{M} \sum_{l=0}^{M-1} T_{l}(z) X\left(z W^{l}\right)
$$

where $W=e^{-j 2 \pi / M}$ and

$$
\begin{aligned}
T_{l}(z) & =\sum_{k=0}^{M-1} F_{k}(z) H_{k}\left(z W^{l}\right) \\
& =\sum_{k=0}^{M-1} J_{k} H_{k}(z) H_{k}\left(z W^{l}\right) .
\end{aligned}
$$

In a perfect-reconstruction system, it is required that $T_{0}$ $=z^{-(L-1)}$ and $T_{l}(z)=0$ for $l \neq 0$. Define the error function $e_{l}(z)$ to be the difference between $T_{l}(z)$ and its desired functions, i.e.,

$$
e_{0}(z)=T_{0}(z)-z^{-(L-1)}, \quad e_{l}(z)=T_{l}(z), l \neq 0
$$

then the objective of the NPR design method is to choose $H_{k}(z)$ such that $e_{l}(z)$ approximate 0 . Moreover, $H_{k}(z)$ must also have good frequency responses. Define the following objective function:

$$
\Phi=\sum_{k=0}^{M-1} \alpha_{k} \phi_{k}+\sum_{l=0}^{M-1} \beta_{l} \hat{e}_{l}
$$

where $\alpha_{k}$ are the weights (chosen by the user) and $\phi_{k}$ are the stopband energies of $H_{k}\left(e^{j \omega}\right)$. Similarly, $\beta_{l}$ are the 
TABLE III

(Design ex. 3) Filter Coefficients of a 8-Channel NPR System. The FILTERS $h_{0}(n), h_{2}(n), \cdots$ ARE SYMMETRIC WHILE $h_{1}(n), h_{3}(n), \cdots$ ARE ANTisYMmetric. FUrThermore, $h_{7-k}(n)=(-1)^{n} h_{k}(n)$. The Aliasing IS $-75.12 \mathrm{db}$. The Stopband Attenuation is $-63 \mathrm{db}$

\begin{tabular}{|c|c|c|c|c|}
\hline$=$ & $h_{0}(n)$ & $h_{2}(n)$ & $h_{4}(n)$ & $h_{6}(n)$ \\
\hline$\overline{0}$ & $E-05$ & -04 & $\overline{E-04}$ & $\overline{E-0}$ \\
\hline 1 & $949578309 E-04$ & $11999673 E-04$ & $-9.6699951958700 E-05$ & $4.3396942046845 E-04$ \\
\hline & & $5 E-05$ & $94843 E-04$ & \\
\hline 3 & $115914 E-04$ & $2355 E-04$ & $4.9934505469239 E-04$ & $2.5407248393889 E-04$ \\
\hline 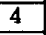 & $9409 E-04$ & $42127547 E-04$ & $6757097 E-04$ & $\overline{322 E-04}$ \\
\hline$F$ & $89 E-04$ & $79 E-04$ & $\overline{0 E-03}$ & -9.4 \\
\hline 6 & $91842 E-04$ & $-9.3747678881810 E-04$ & $60847 E-04$ & $7746 E-03$ \\
\hline 7 & $2 E-04$ & $9 \overline{E-03}$ & $77 E-03$ & $5 E-04$ \\
\hline 8 & $0 E-04$ & $\overline{3 E-04}$ & $9 E-04$ & $69 E-04$ \\
\hline 9 & $36 E-03$ & $36 E-05$ & $92 \bar{E}-06$ & $377 E-05$ \\
\hline$\overline{10}$ & $3 E-03$ & $E-03$ & $\overline{8 E-04}$ & $8 E-04$ \\
\hline 11 & $E-03$ & $E-03$ & $2 \overline{2 E-03}$ & $2 E-03$ \\
\hline 12 & $E-03$ & $\overline{E-03}$ & $4 E-03$ & $7 \overline{7}-03$ \\
\hline 5 & $E-03$ & $E-03$ & $\overline{6 E-03}$ & $\overline{9 E-03}$ \\
\hline 14 & & $\overline{E-03}$ & $\overline{E-03}$ & $2 E-04$ \\
\hline 15 & $E-03$ & $E-03$ & $2 \bar{E}-\overline{03}$ & $8 E-03$ \\
\hline 16 & $E-03$ & $E-02$ & $E-02$ & $2 E-02$ \\
\hline 17 & $E-03$ & $E-02$ & $0 E-03$ & $9 E-02$ \\
\hline 18 & $E-03$ & $E-03$ & $2 E-02$ & $\overline{44 E-02}$ \\
\hline 19 & $E-03$ & $E-02$ & $6 \overline{E-03}$ & $E-03$ \\
\hline 20 & $E-03$ & $E-03$ & $E-03$ & $3 E-03$ \\
\hline 21 & $E-04$ & $E-04$ & $E-03$ & $6 E-03$ \\
\hline 22 & $E-03$ & $E-04$ & $E-04$ & $1 E-03$ \\
\hline 23 & $\overline{E-02}$ & $E-03$ & $\overline{6 E-03}$ & $3 E-03$ \\
\hline 24 & $\overline{E-02}$ & $E-03$ & $\overline{\bar{E}-03}$ & $1 E-03$ \\
\hline$\overline{25}$ & -02 & -02 & $E-02$ & $8 E-03$ \\
\hline 26 & $\overline{E-02}$ & $E-02$ & $\overline{E-02}$ & $0 E-02$ \\
\hline 27 & $4 E-02$ & $\overline{1 E-02}$ & $\overline{5 E-02}$ & $43 E-02$ \\
\hline$\frac{28}{28}$ & $\overline{E-03}$ & & $3 E-02$ & $1 E-02$ \\
\hline 29 & $E-02$ & $\overline{02}$ & $\overline{E-02}$ & $7 E-02$ \\
\hline 30 & $4 E-02$ & $9 E-02$ & $5 E-02$ & $37 E-02$ \\
\hline 31 & $E-02$ & $E-02$ & -02 & \\
\hline 32 & $E-02$ & & & 124645 \\
\hline 33 & 2695 & 943894 & $E-02$ & 3217950 \\
\hline 2 & & & & \\
\hline 35 & 57 & & & \\
\hline
\end{tabular}

weights for $\hat{e}_{l}$, where

$$
\hat{e}_{l}=\left|\max _{n} e_{l}(n)\right|^{2}
$$

In order to simplify and reduce the number of variables in the design problem, we impose the condition that the filters also satisfy the pairwise mirror image property, i.e., $H_{M-1-k}(z)=H_{k}(-z)$. We have provided three design examples in this section. In the tables, the filters with even indices are symmetric and those with odd indices are antisymmetric. Fig. 10 shows the magnitude response of an 8-channel linear phase NPR system (Design ex. 3). The coefficients have been tabulated in Table III. Similarly, Tables IV and $\mathrm{V}$ give the impulse response coefficients for 4-channel NPR system (Design ex. 4) and 12-channel NPR system (Design ex. 5). In each table, the aliasing is defined to be the quantity $20 \log _{10}\left[\max _{\omega, l}\left|T_{l}\left(e^{j \omega}\right)\right|\right]$, where $T_{l}(z)$ is as in (7.2). The minimum stop-band attenuations of the analysis filters are also indicated in the table captions.
TABLE IV

(Design ex. 4) Filter Coffficients of a 4-Channel NPR System. The Filters $h_{0}(n), h_{2}(n)$ ARE SYMMETRIC WhILE $h_{1}(n)$, AND $h_{3}(n)$ ARE ANTISYMMETRIC. FURTHERMORE, $h_{3-k}(n)=(-1)^{n} h_{k}(n)$. THE ALIASING IS $-70.97 \mathrm{db}$. The Stopband Attenuation is $-59 \mathrm{db}$

\begin{tabular}{c||r|r}
\hline$n$ & $h_{0}(n)$ & $h_{2}(n)$ \\
\hline \hline 0 & $-5.5444726422155 E-04$ & $2.3771721828281 E-04$ \\
\hline 1 & $1.9839695392787 E-05$ & $-1.4383078227793 E-03$ \\
\hline 2 & $1.0331185134746 E-03$ & $2.2053690376212 E-04$ \\
\hline 3 & $3.1253201831343 E-03$ & $-8.7972077858063 E-04$ \\
\hline 4 & $3.2525521320976 E-03$ & $5.4151997528059 E-03$ \\
\hline 5 & $-1.6021091462862 E-03$ & $2.7381741936965 E-03$ \\
\hline 6 & $-1.0139991022229 E-02$ & $-2.2201801616551 E-02$ \\
\hline 7 & $-1.4657353520819 E-02$ & $1.4530811545905 E-02$ \\
\hline 8 & $-5.8631726134028 E-03$ & $1.1123987448548 E-02$ \\
\hline 9 & $1.7471272374347 E-02$ & $1.4906518746196 E-03$ \\
\hline 10 & $4.1126463410573 E-02$ & $-7.9151202613239 E-03$ \\
\hline 11 & $3.9606212551725 E-02$ & $-7.8671269271711 E-02$ \\
\hline 12 & $-7.5685271761424 E-03$ & 0.13152350759896 \\
\hline 13 & $-9.7873310743775 E-02$ & $4.3220116924369 E-02$ \\
\hline 14 & -0.19976296359216 & -0.26641530036823 \\
\hline 15 & -0.26742606716914 & 0.16653767332615 \\
\hline
\end{tabular}


TABLE $V$

(Design ex. 5) Filter Coefficients of a 12-Channel NPR System. The FILTERS $h_{0}(n), h_{2}(n), \cdots$ ARE SYMMETRIC WHILE $h_{1}(n), h_{3}(n), \cdots$ ARE

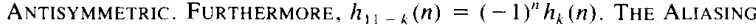
is $-56.71 \mathrm{db}$. The Stopband Attenuation is $-38 \mathrm{db}$

\begin{tabular}{|c|c|c|c|}
\hline$n$ & $h_{6}(n)$ & $h_{8}(n)$ & $h_{10}(n)$ \\
\hline$\overline{0}$ & $\overline{8 E-04}$ & $E-0 \overline{3}$ & $\overline{E-03}$ \\
\hline & $1.1096974566709 E-04$ & $1.0253944460934 \bar{E}-03$ & $2.1085447394946 E-0$ \\
\hline & $\overline{E-03}$ & $E-04$ & $7 E-03$ \\
\hline 3 & $E-03$ & $\overline{00 E-03}$ & $229565423 E-03$ \\
\hline 4 & $-2.5121231147945 E-03$ & $4.1675889148922 E-03$ & $2.4889411486735 E-03$ \\
\hline & $072582 E-\overline{03}$ & $-2.7248841713913 E-03$ & $3798278089 E-03$ \\
\hline 6 & $\overline{E E-03}$ & $\overline{433675 \bar{E}-03}$ & $5 E-03$ \\
\hline 7 & $-5.2936370785786 E-03$ & $5.6963880713784 E-03$ & $3.6307082282229 E-03$ \\
\hline o & $-2.6135816627454 E-03$ & $-4 . \overline{3595342271162 E-03}$ & $\overline{2940151865 E-\overline{03}}$ \\
\hline 9 & $E-03$ & $2 E-04$ & $E E-$ \\
\hline 10 & $E-04$ & $2.5111441001857 E-03$ & $72032295 E-03$ \\
\hline 11 & $6 \bar{E}-\overline{04}$ & $5.6629726333247 E-04$ & $826597137 E-04$ \\
\hline 12 & $E-05$ & $\overline{0 E-04}$ & $559292 E-03$ \\
\hline$\overline{13}$ & $E-\mathbf{0 3}$ & $E-03$ & -1.2 \\
\hline 14 & $69297214 \bar{E}-03$ & $8 E-02$ & $23921 E-03$ \\
\hline 15 & $3 E-02$ & $-1.0822522726306 E-02$ & $912060 E-03$ \\
\hline 16 & $\overline{E-02}$ & $5 E-03$ & $\overline{23 E-02}$ \\
\hline 17 & $\overline{E-02}$ & $7 \bar{E}-02$ & $20426 E-02$ \\
\hline 18 & $\overline{3 E-02}$ & $-3.4107229799077 E-02$ & $\overline{3808277 E-02}$ \\
\hline 19 & $E-02$ & $6 E-03$ & $\overline{97 E-02}$ \\
\hline 20 & $\overline{E-02}$ & $E-02$ & $5 E-02$ \\
\hline 21 & $\overline{E-02}$ & $16 E-02$ & $188254 E-02$ \\
\hline 22 & $E-02$ & $E-02$ & $1 E-02$ \\
\hline 23 & -03 & $E-02$ & $83790 E-02$ \\
\hline 24 & -02 & $E-02$ & $\overline{3 E-02}$ \\
\hline 25 & $E-02$ & $E-02$ & $7 \overline{E-02}$ \\
\hline 26 & $\overline{E-02}$ & $E-03$ & $1 E-02$ \\
\hline 27 & $E-02$ & $3 E-02$ & $46 E-02$ \\
\hline 28 & $\overline{E-02}$ & $\overline{E-01}$ & $2 E-02$ \\
\hline 29 & $E-02$ & $\overline{E-02}$ & $2 E-c$ \\
\hline$n$ & $h_{0}(n)$ & $h_{2}$ & $h_{4}(n)$ \\
\hline 0 & $\overline{D E-04}$ & $1 E-0 \overline{3}$ & $38 E-03$ \\
\hline 1 & $E-04$ & $E-03$ & $6 E-04$ \\
\hline 2 & $\overline{E-04}$ & $4 \bar{E}-03$ & $37 E-03$ \\
\hline 3 & $-\overline{03}$ & $E-04$ & $1 E-03$ \\
\hline . & -03 & $2 E-03$ & $2 E-03$ \\
\hline 0 & -0 & $9 E-03$ & $6 E-03$ \\
\hline 6 & $-\overline{03}$ & $\overline{7} \bar{E}-03$ & $\overline{5 E-03}$ \\
\hline 7 & & & $\overline{9 E-03}$ \\
\hline 0 & -03 & $\bar{E}-04$ & $\overline{4 E-03}$ \\
\hline 2 & -04 & $E-03$ & $0 E-03$ \\
\hline 10 & $-\overline{03}$ & $\overline{E-03}$ & $5 E-03$ \\
\hline & & -05 & $E-04$ \\
\hline & & (2) & $7 E$ \\
\hline 13 & -02 & $E-03$ & $9 E-03$ \\
\hline 14 & & & $4 E-03$ \\
\hline 15 & & -02 & $E-03$ \\
\hline & & $E-02$ & $2 F$ \\
\hline 17 & & & $\overline{4} \bar{E}-0 \overline{2}$ \\
\hline 18 & & -02 & $2 E-02$ \\
\hline $1 !$ & & & 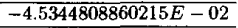 \\
\hline & & & $2 E-02$ \\
\hline 21 & $3-02$ & $8-02$ & $4 E-02$ \\
\hline 22 & & -02 & $\overline{E-02}$ \\
\hline 23 & & & $4 F=02$ \\
\hline 24 & & & \\
\hline 2 & $\overline{E-02}$ & $\overline{4}-0 \overline{2}$ & $55 E-02$ \\
\hline 27 & & & $\overline{E-02}$ \\
\hline 2 & & & \\
\hline & & & \\
\hline & 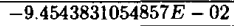 & & \\
\hline
\end{tabular}

\section{Conclusions}

In this paper, we studied in detail the theory, factorizations and designs of linear phase paraunitary systems. In Section II, we proved several results on linear phase paraunitary systems, which we used subsequently. Next we addressed the problem of designing linear phase paraunitary systems for an even number of channels $M$. We showed that such systems could be designed by a cascade structure which was proved to be minimal. The resulting filters are structurally linear phase paraunitary, i.e., these properties are preserved inspite of coefficient quantization. Moreover, we showed the completeness of this structure, i.e., all linear phase paraunitary systems satisfying (2.1) can be generated simply by manipulating the coefficients of this cascade structure. Next, we imposed the further condition on the filters that they satisfy the pairwise mirror-image property in the frequency domain. The resulting structure has much fewer multipliers, which is useful for optimization. To summarize therefore, for these filter banks the following properties are guaranteed structurally, i.e., inspite of quantization of the multipliers (angles):

- The filter bank is paraunitary, and therefore gives perfect reconstruction.

- The analysis and synthesis filters are time-reversed versions of each other.

- The analysis and synthesis filters are all linear phase.

- The filters in the analysis and synthesis banks both satisfy the pairwise mirror-image property in the frequency domain.

Next, we extended this analysis to the case of filter banks with an odd number of channels $M$. In particular, we showed two ways by which such systems could be realized. One was based on a factorization approach, and the other involved designing larger systems by successively combining smaller systems in a certain manner.

It is interesting to note that the linear phase property along with the paraunitary condition implies that the analysis and synthesis banks are identical, upto a multiplier of \pm 1 on some of the filters, i.e., $F_{i}(z)= \pm H_{i}(z)$.

We then considered two applications of the theory. The first was in designing symmetric and antisymmetric $M$-band wavelets which are also orthonormal. We also discussed the regularity condition in this context, and derived conditions on the factorization proposed so that the resulting wavelets had at least one vanishing moment. The second application we considered was in subband coding. From the data presented for lowpass speech, we conclude that the linear phase paraunitary systems with filters of small length give good coding gains. With the other special features of the filters mentioned before, we conclude that this structure is a good candidate for use in practical subband coding systems.

APPENDIX A

Consider the matrix $J_{M}$ where $M$ is even. The eigenvalues of this matrix are \pm 1 , and the corresponding eigenvectors are the symmetric and antisymmetric vectors of size $M$. We will refer to the two eigenspaces of the matrix $J_{M}$ as the symmetric and antisymmetric eigenspaces $\mathcal{E}_{s}$ and $\mathcal{E}_{a}$ respectively. The basis for $\mathcal{E}_{s}$ could be the set of vectors $s_{i}, i=0, \cdots(M / 2)-1$, where all 
elements of the vectors $s_{i}$ are zero, except $s_{i}(i)=s_{i}(M-$ $1-i)=1$. Similarly, a basis for $\varepsilon_{a}$ could be the set of vectors $a_{i}, i=0, \cdots(M / 2)-1$, where all elements of the vectors $a_{i}$ are zero, except $a_{i}(i)=-a_{i}(M-1-i)$ $=1$. Also, since the matrix $\boldsymbol{J}_{M}$ is symmetric, the eigenvectors span the whole space, and $\mathcal{E}_{s}$ and $\mathcal{E}_{a}$ form a direct sum for the whole space. Now, consider any vector $y$. It can always be written as $\boldsymbol{y}=\boldsymbol{u}+\boldsymbol{v}$, where $\boldsymbol{u} \in \mathcal{E}_{s}$ and $\boldsymbol{v}$ $\in \mathcal{E}_{a}$. Let $\boldsymbol{y}$ be orthogonal to its own flipped version, i.e.,

$$
\boldsymbol{y}^{T} \boldsymbol{J}_{M} \boldsymbol{y}=0 \text {. }
$$

Hence we get,

$$
(\boldsymbol{u}+\boldsymbol{v})^{T} \boldsymbol{J}_{M}(\boldsymbol{u}+\boldsymbol{v})=0 .
$$

Noting that $\boldsymbol{u}^{T} \boldsymbol{J}_{M} \boldsymbol{u}=\boldsymbol{u}^{T} \boldsymbol{u}, \boldsymbol{v}^{T} \boldsymbol{J}_{M} \boldsymbol{v}=-\boldsymbol{v}^{T} \boldsymbol{v}$ and $\boldsymbol{u}^{T} J_{M} \boldsymbol{v}$ $=0$, the above equation reduces to $\boldsymbol{u}^{T} \boldsymbol{u}=\boldsymbol{v}^{T} \boldsymbol{v}$. Hence, the norm of the projections in the two eigenspaces has to be equal. We say therefore that the vector $y$ which satisfies (A.1) is "balanced" over the two eigenspaces (or simply "balanced").

As noted above, the eigenvectors of the matrix $\boldsymbol{J}$ are symmetric and antisymmetric vectors (have linear phase). Furthermore, these eigenvectors are orthonormal. Hence, one would expect the eigenstructure of the $J$ matrix to play a role in the synthesis of linear phase orthonormal systems.

\section{APPENDIX B}

From (3.28) we have $W^{T} f_{m+1}^{T}(0) J_{M} f_{m+1}(0) W=0$, for any matrix $W$. This means that the columns of $f_{m+1}(0) W$ are balanced. Let the matrix $W$ be so chosen that the first $r$ columns of the matrix $f_{m+1}(0) W$ form an orthonormal basis for the columns of matrix $f_{m+1}(0)$. Denote these $r$ vectors as $x_{i}, i=1, \cdots, r$. Hence, the vectors $x_{i}$ are balanced and orthonormal, i.e., $\boldsymbol{x}_{i}^{T} \boldsymbol{x}_{j}=0$. Let $x_{i}=u_{i}^{\prime}+v_{i}^{\prime}$, where $u_{i}^{\prime} \in \mathcal{E}_{s}$ and $v_{i}^{\prime} \in \mathcal{E}_{a}$. Therefore, $\left(\boldsymbol{u}_{i}^{\prime}+\boldsymbol{v}_{i}^{\prime}\right)^{T}\left(\boldsymbol{u}_{j}^{\prime}+\boldsymbol{v}_{j}^{\prime}\right)=0$, which simplifies to

$$
\boldsymbol{u}_{i}^{\prime T} \boldsymbol{u}_{j}^{\prime}+\boldsymbol{v}_{i}^{\prime T} \boldsymbol{v}_{j}^{\prime}=0 .
$$

Since the vectors $\boldsymbol{x}_{i}$ are balanced, i.e., $\boldsymbol{x}_{i}^{T} \boldsymbol{J}_{M} \boldsymbol{x}_{j}=0$, we have $\left(u_{i}^{\prime}+v_{i}^{\prime}\right)^{T} J_{M}\left(u_{j}^{\prime}+v_{j}^{\prime}\right)=0$, simplifying which we get

$$
\boldsymbol{u}_{i}^{\prime T} \boldsymbol{u}_{j}^{\prime}=\boldsymbol{v}_{i}^{\prime T} \boldsymbol{v}_{j}^{\prime}
$$

Equations (B.1) and (B.2) together imply that $\boldsymbol{u}_{i}^{\prime T} \boldsymbol{u}_{j}^{\prime}=$ 0 , and $\boldsymbol{v}_{i}^{\prime T} \boldsymbol{v}_{j}^{\prime}=0$. The vectors $u_{i}^{\prime}, i=1, \cdots, r$ and $\boldsymbol{v}_{i}^{\prime}, i=1, \cdots, r$ therefore form orthonormal bases for $r$-dimensional subspaces of $\varepsilon_{s}$ and $\varepsilon_{a}$ respectively. In $\varepsilon_{s}$, there exist $p=M / 2-r$ orthogonal vectors $\boldsymbol{u}_{i}^{\prime}, i=r+$ $1, \cdots, M / 2$ which are also orthogonal to the previously mentioned set of $r$ vectors $\boldsymbol{u}_{i}^{\prime}, i=1, \cdots, r$. Similarly, in $\mathcal{E}_{a}$, there exist $p=M / 2-r$ orthogonal vectors $v_{i}^{\prime}, i$ $=r+1, \cdots, M / 2$ which are also orthogonal to the previously mentioned set of $r$ vectors $v_{i}^{\prime}, i=1, \cdots, r$. Now using these additional $p$ orthonormal vectors from $\mathcal{E}_{s}$ and $\varepsilon_{a}$, we can form $p$ orthonormal, balanced vectors. With this construction, it can be verified that the set of $M / 2$ vectors $\boldsymbol{x}_{i}=\boldsymbol{u}_{i}^{\prime}+\boldsymbol{v}_{i}^{\prime}, i=1, \cdots, M / 2$ satisfies the following properties:

1) They are orthonormal and balanced.

2) They are also orthonormal to the flipped versions of each other. Hence, if $\boldsymbol{X}^{T}$ is the matrix of size $M / 2 \times M$ which has these vectors as its rows, this matrix satisfies the property $X^{T} J_{M} f_{m+1}(0)=0$.

\section{REFERENCES}

[1] R. Crochiere and L. Rabiner, Multirate Digital Signal Processing. Englewood Cliffs, NJ: Prentice-Hall, 1983.

[2] J. W. Woods and S. D. O'Neil, "Subband coding images," IEEE Trans. Acoust., Speech, Signal Processing, vol. ASSP-34, pp. 12781299. Oct. 1986.

[3] P. P. Vaidyanathan, "Multirate digital filters, filter banks, polyphase networks, and applications: A tutorial," Proc. IEEE, vol. 78, Jan. 1990.

[4] W. A. Pearlman, "Performance bounds for subband coding," in J. Woods, Ed., Subband Image Coding. Boston, MA: Kluwer, 1991, ch. 1 .

[5] M. J. T. Smith and T. P. Barnwell, III, "A procedure for designing exact reconstruction filter banks for tree-structured subband coders,' Proc, ICASSP, San Diego, CA, Mar. 1984.

[6] F. Mintzer, "Filters for distortion-free two-band multirate filter banks,"' IEEE Trans. Acoust., Speech, Signal Processing, pp. 626630, June 1985.

[7] M. Vetterli, "Filter banks allowing for perfect reconstruction," Signal Processing, vol. 10, pp. 219-244, Apr. 1986.

[8] P. P. Vaidyanathan, "Theory and design of $M$-channel maximally decimated filters with arbitrary $M$, having perfect reconstruction property," IEEE Trans. Acoust., Speech, Signal Processing, vol. ASSP-35, pp. 476-492, Apr. 1987.

[9] M. Vetterli, "A theory of multirate filter banks," IEEE Trans. Acoust., Speech, Signal Processing, vol. ASSP-35, pp. 356-372, Mar. 1987.

[10] P. P. Vaidyanathan, Multirate Systems and Filter Banks. Englewood Cliffs, NJ; Prentice-Hall, 1993.

[11] H. S. Malvar, "Lapped transforms for efficient transform/subband coding,' IEEE Trans. Acoust., Speech, Signal Processing, vol. 38, June 1990.

[12] K. Nayebi, T. Barnwell, and M. Smith, "A general time domain analysis and design framework for exact reconstruction FIR analysis/ synthesis filter banks," Proc. ISCAS, New Orleans, LA, May 1990, pp. 2022-2025.

[13] O. Rioul and $\mathrm{M}$. Vetterli, "Wavelets and signal processing," IEEE Signal Processing Mag., vol. 8, pp. 14-38, Oct. 1991.

[14] V. Belevitch, Classical Network Theory. San Francisco, CA: Holden Day, 1968.

[15] A. Soman and P. P. Vaidyanathan, "Paraunitary filter banks and wavelet packets,"' Proc. ICASSP, San Francisco, CA, 1992, pp. 397 400 .

[16] P. P. Vaidyanathan and Z. Doğanata, "The role of lossless systems in modern digital signal processing: A tutorial," IEEE Trans. Education, Aug. 1989.

[17] A. Soman and P. P. Vaidyanathan, "Coding gain in paraunitary analysis synthesis systems," IEEE Trans. Acoust., Speech, Signal Processing, vol. 41, pp. 1824-1825, May 1993.

[18] N. S. Jayant and P. Noll, Digital Coding of Waveforms-Principles and Applications to Speech and Video. Englewood Cliffs, NJ: Prentice-Hall, 1984.

[19] I. Daubechies, "Ten lectures on wavelets,"' SIAM CBMS series, Apr. 1992.

[20] H. Zou and A. H. Tewfik, "Discrete orthogonal $M$-band wavelet decompositions," Proc. ICASSP, San Francisco, CA, Mar. 1992.

[21] A. Tewfik and M. Kim, "Fast multiscale signal processing algorithms," Proc. ICASSP, San Francisco, CA, Mar. 1992, pp. 373376 . 
[22] M. Vetterli and D. Le Gall, "Analysis and design of perfect reconstruction filter banks satisfying symmetry constraints," Proc. 22nd Conf. Informat. Sci. Syst., Princeton, NJ, Mar. 1988.

[23] T. Nguyen and P. P. Vaidyanathan, "Two channel perfect reconstruction FIR QMF structures which yield linear-phase analysis filters,"' IEEE Trans. Acoust., Speech, Signal Processing, vol. 37, pp. 676-690, May 1989.

[24] J. Princen and A. Bradley, "Analysis/synthesis filter bank design based on time-domain aliasing cancellation," IEEE Trans. Acoust., Speech, Signal Processing, vol. ASSP-36, pp. 1153-1161, Oct. 1986.

[25] H. S. Malvar and D. Staelin, "The LOT: Transform coding without blocking effects," IEEE Trans. Acoust., Speech, Signal Processing, vol. 37, pp. 553-559, Apr. 1989.

[26] M. Vetterli and D. Le Gall, "Perfect reconstruction filter banks: Some properties and factorizations," IEEE Trans. Acoust., Speech, Signal Processing, vol. 37, pp. 1057-1071, July 1989.

[27] M. Bellanger, G. Bonnerot, and M. Coudreuse, "Digital filtering by polyphase network: Application to sample rate alteration and filte banks," IEEE Trans. Acoust., Speech, Signal Processing, vol. ASSP24, pp. 109-114, Apr. 1976

[28] P. Cassereau, "A new class of orthogonal transforms for image processing," M.S. thesis, Dept. Elec. Eng. Comput. Sci., Massachusetts Inst. Tech., Cambridge, MA, May 1985.

[29] F. Murnaghan, The Unitary and Rotation Groups. Washington D.C.: Spartan Books, 1962.

[30] R. Horn and C. Johnson, Matrix Analysis. Cambridge, MA: Cambridge Univ. Press, 1985.

[31] T. Nguyen and P. P. Vaidyanathan, "Maximally decimated perfectreconstruction filter banks with pairwise mirror-image analysis (and synthesis) frequency responses," IEEE Trans. Acoust., Speech, Signal Processing, vol. ASSP-36, pp. 693-706, May 1988.

[32] I. Daubechies, "Orthonormal bases of compactly supported wavelets," Commun. Pure Appl. Math., vol. 4, pp. 909-996, Nov. 1988

[33] R. Coifman, Y. Meyer, S. Quake, and V. Wickerhauser, "Signal processing with wavelet packets," Numerical Algorithms Research Group, Yale University, New Haven, CT, 1990.

[34] S. Mallat, "Multifrequency channel decomposition of images and wavelet models," IEEE Trans. Acoust., Speech, Signal Processing, vol. 37, pp. 2091-2110, 1989.

[35] M. Antonini, M. Barlaud, P. Mathieu, and I. Daubechies, "Image coding using vector quantization in wavelet transform domain, "Proc. ICASSP, Albuquerque, NM, 1990, pp. 2297-2300.
[36] R. Gopinath and C. Burrus, "Wavelet transforms and filter banks," in C. H. Chui, Ed., Wavelets and Applications. New York: Academic Press, 1991.

[37] S. Mallet, "Multiresolution approximations," Trans. Amer. Math Soc., Sept. 1989.

[38] P. Heller and H. Resnikoff, "Regular $M$-band wavelets and applications," Proc, ICASSP, Minneapolis, MN, Apr. 1993

[39] W. Lawton, "Tight frames of compactly supported affine wavelets, " J. Math. Phys., Aug. 1990.

Anand K. Soman (S'91) for a photograph and biography, see page 1835 of the May 1993 issue of this TransaCtions.

P. P. Vaidyanathan (S'80-M'83-SM'88-F'91) for a photograph and biography, see page 1835 of the May 1993 issue of this TransaCtions.



Truong Q. Nguyen (S;85-M'89) was born in Saigon, Vietnam, on November 2,1962 . He received the B.S. (Hons.), M.S., and Ph.D. degrees from the California Institute of Technology, Pasadena, in electrical engineering in 1985, 1986, and 1989, respectively.

$\mathrm{He}$ is currently with Lincoln Laboratory, Massachusetts Institute of Technology, Cambridge. During the academic year 1986-1987, he was a recipient of a fellowship from Aerojet Dynamics, for advanced studies. His main research interests are in digital signal processing, multirate systems and applications, filter design, ultrasonics nondestructive evaluation and adaptive array processing.

Dr. Nguyen received the IEEE Signal Processing's 1992 Paper Award (Image and Multidimensional Processing area) for the paper with P. P. Vaidyanathan on linear-phase perfect-reconstruction filter banks (TRANSaction on Signal Processing, Mar. 1990). He is a member of Tau Beta $\mathrm{Pi}$, Eta Kappa Nu and Sigma Xi. 